\title{
Cooperativity of imprinted genes inactivated by acquired chromosome 20 q deletions
}

\author{
Athar Aziz, 1,2 E. Joanna Baxter,1,2,3 Carol Edwards, ${ }^{4}$ Clara Yujing Cheong, ${ }^{5}$ Mitsuteru Ito, 4 \\ Anthony Bench, ${ }^{3}$ Rebecca Kelley, ${ }^{1,2}$ Yvonne Silber, ${ }^{1,2}$ Philip A. Beer,, ${ }^{1,2,3}$ Keefe Chng, ${ }^{5}$ \\ Marilyn B. Renfree, ${ }^{6}$ Kirsten McEwen, ${ }^{4}$ Dionne Gray,, Jyoti Nangalia, ${ }^{1,2,3}$ Ghulam J. Mufti,7 \\ Eva Hellstrom-Lindberg, ${ }^{8}$ Jean-Jacques Kiladjian,9,10 Mary Frances McMullin, ${ }^{11}$ \\ Peter J. Campbell, ${ }^{2,3,12}$ Anne C. Ferguson-Smith, ${ }^{4}$ and Anthony R. Green ${ }^{1,2,3}$ \\ ${ }^{1}$ Cambridge Institute for Medical Research and Wellcome Trust/MRC Stem Cell Institute and 2Department of Hematology, \\ University of Cambridge, Cambridge, United Kingdom. ${ }^{3}$ Department of Hematology, Addenbrooke's Hospital, Cambridge, United Kingdom. \\ ${ }^{4}$ Department of Physiology, Development, and Neuroscience, University of Cambridge, Cambridge, United Kingdom. \\ ${ }^{5} A^{*}$ STAR Singapore Institute for Clinical Sciences, Brenner Centre for Molecular Medicine, Singapore. ${ }^{6}$ Department of Zoology, \\ University of Melbourne, Melbourne, Victoria, Australia. 'Department of Hematological Medicine, King's College London, London, United Kingdom. \\ ${ }^{8}$ Karolinska Institute, Department of Medicine, Division of Hematology, Karolinska University Hospital Huddinge, Stockholm, Sweden. \\ ${ }^{9}$ Hopital Saint-Louis, AP-HP, Centre d'Investigations Cliniques, Paris, France. ${ }^{10}$ Université Paris 7 Denis Diderot, Paris, France. \\ ${ }^{11}$ Center for Cancer Research and Cell Biology, Queen's University, Belfast, United Kingdom. \\ ${ }^{12}$ Wellcome Trust Sanger Institute, Hinxton, Cambridge, United Kingdom.
}

\begin{abstract}
Large regions of recurrent genomic loss are common in cancers; however, with a few well-characterized exceptions, how they contribute to tumor pathogenesis remains largely obscure. Here we identified primaterestricted imprinting of a gene cluster on chromosome 20 in the region commonly deleted in chronic myeloid malignancies. We showed that a single heterozygous $20 \mathrm{q}$ deletion consistently resulted in the complete loss of expression of the imprinted genes $L 3 M B T L 1$ and $S G K 2$, indicative of a pathogenetic role for loss of the active paternally inherited locus. Concomitant loss of both L3MBTL1 and SGK2 dysregulated erythropoiesis and megakaryopoiesis, 2 lineages commonly affected in chronic myeloid malignancies, with distinct consequences in each lineage. We demonstrated that L3MBTL1 and SGK2 collaborated in the transcriptional regulation of MYC by influencing different aspects of chromatin structure. L3MBTL1 is known to regulate nucleosomal compaction, and we here showed that SGK2 inactivated BRG1, a key ATP-dependent helicase within the SWI/ SNF complex that regulates nucleosomal positioning. These results demonstrate a link between an imprinted gene cluster and malignancy, reveal a new pathogenetic mechanism associated with acquired regions of genomic loss, and underline the complex molecular and cellular consequences of "simple" cancer-associated chromosome deletions.
\end{abstract}

\section{Introduction}

Many human malignancies harbor large-scale regions of genetic loss that may be evident as monosomy, interstitial deletion, or acquired uniparental disomy (UPD), with particular changes often recurrent in specific tumor types $(1,2)$. UPD may be associated with gain-of-function mutations that become homozygous following mitotic recombination (e.g., JAK2 ${ }^{V 617 F}$, refs. 3-6). Some chromosomal deletions mark the site of a tumor suppressor gene that is inactivated on the normal chromosome (e.g., RB and TP53), whereas others are thought to reflect haploinsufficiency of at least 1 target gene (7-9). However, in most cases, our understanding of the underlying mechanisms is incomplete or absent.

Chronic myeloid malignancies are associated with deletions of 20q (10-12). The 20q minimal deleted region spans $1.9 \mathrm{Mb}$ and contains multiple genes, including L3MBTL1 (13), a transcriptional repressor (14-16) homologous to the Drosophila tumor-suppressor gene lethal(3) malignant brain tumor $(17,18)$. L3MBTL1 binds to mono- and dimethyl lysine residues through its MBT repeats $(19,20)$, and creates a compacted chromatin structure $(16$,

Authorship note: Athar Aziz and E. Joanna Baxter are co-first authors. Anne C. Ferguson-Smith and Anthony R. Green are co-senior authors. Conflict of interest: The authors have declared that no conflict of interest exists. Citation for this article: J Clin Invest. 2013;123(5):2169-2182. doi:10.1172/JCI66113.
21). Its biological functions are largely unclear, but it is reported to repress E2F target genes (16), inhibit RUNX1 expression (15), and modulate DNA replication and repair (22). Knockdown and overexpression studies in primary $\mathrm{CD}_{3} 4^{+}$cells and in hematopoietic cell lines have indicated that $L 3 M B T L 1$ restricts erythroid differentiation (22). However, acquired mutations in L3MBTL1 have not been found in primary cells from myelodysplastic syndrome (MDS) or myeloproliferative neoplasm (MPN) patients with or without a $20 \mathrm{q}$ deletion $(13,23)$, and transcript levels of L3MBTL1 were reported to be unaffected in primary cells from patients with a $20 \mathrm{q}$ deletion (13)

We have previously demonstrated that $L 3 M B T L 1$ is imprinted in humans, but surprisingly not in mice (Mus musculus) $(24,25)$. Imprinted genes function in the control of prenatal growth, in the development of particular organ systems, including the placenta, and in the postnatal control of metabolic and neural processes $(26,27)$. Loss of imprinting has frequently been found in cancers and is associated with altered patterns of expression that characterize the tumorigenic state (28). Inherited deletions influencing long-range epigenetic control at imprinted loci are associated with increased tumor incidence, particularly in the context of Beckwith-Wiedemann syndrome $(29,30)$, but imprinted loci have not been identified as direct targets of acquired tumor-associated genomic changes. 
A
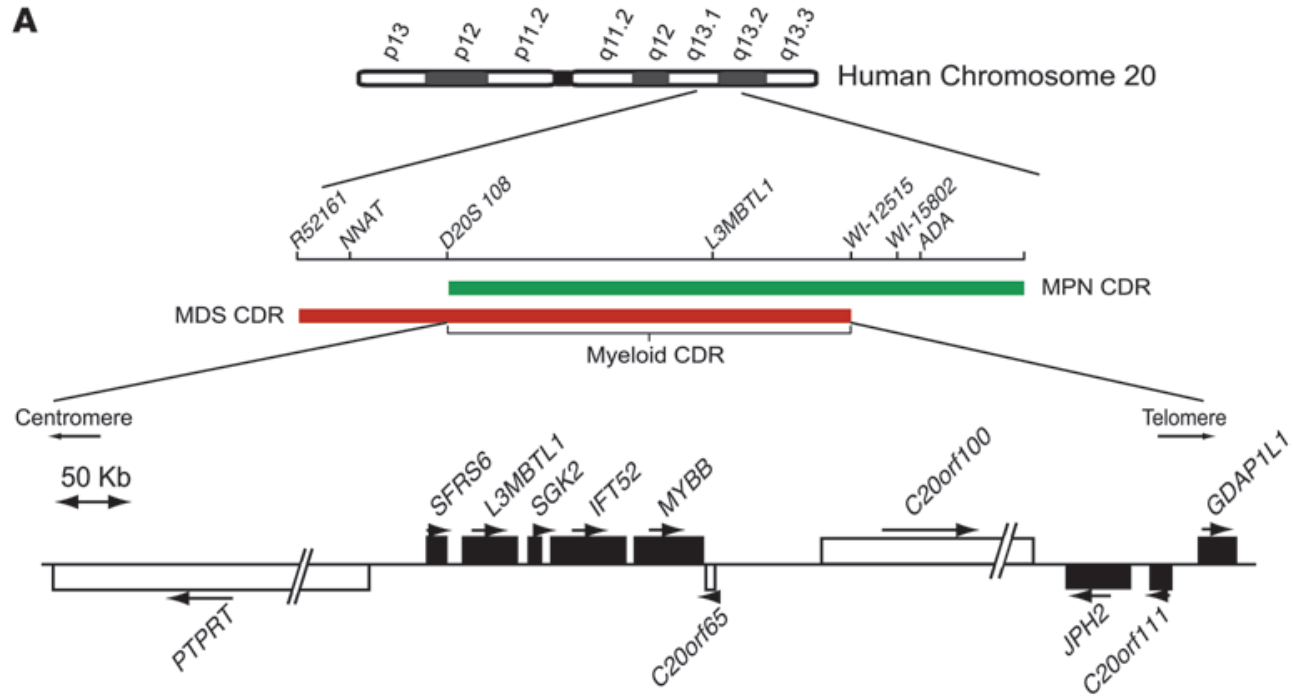

B

\section{L3MBTL1}

SGK2

ATTG ${ }_{T}^{C} A C C$ T
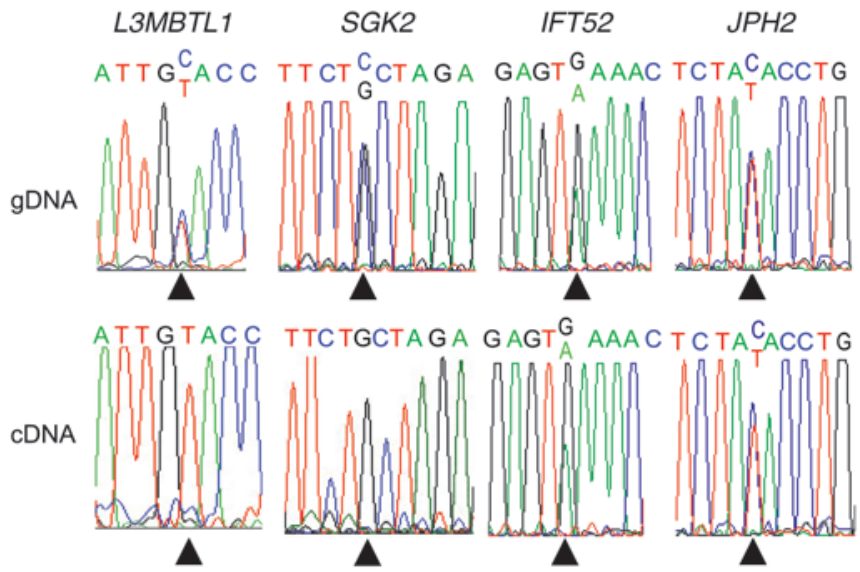

$\mathrm{T}$ cells

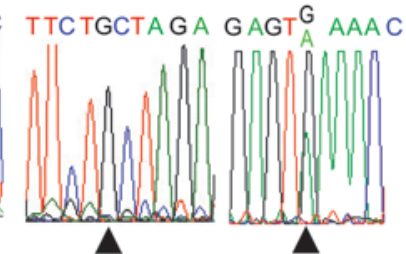

T cells

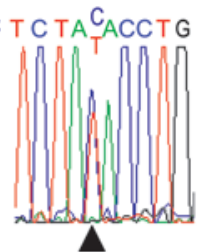

Grans
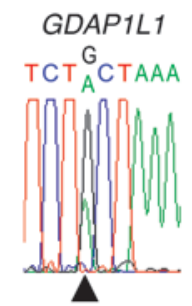

TCTGCTAAA

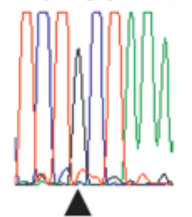

Erythroblasts

\section{Figure 1}

L3MBTL1, SGK2, and GDAP1L1 constitute a novel imprinting cluster. (A) 20q12 region, with MPN and MDS CDRs and the overlapping myeloid CDR, as previously defined by Bench et al. (13). Genes in black are expressed in hematopoietic cells; genes in white are not. (B) Monoallelic expression of L3MBTL1, SGK2, and GDAP1L1 in hematopoietic cells. Grans, granulocytes; gDNA, genomic DNA. (C) Family studies demonstrated that SGK2 and GDAP1L1 were expressed from the paternally derived allele in $T$ cells and erythroblasts, respectively. The respective cell types were chosen because they express readily detectable levels of SGK2 and GDAP1L1. The expressed allele is indicated by an arrowhead. See Supplemental Figure 1 for other families.
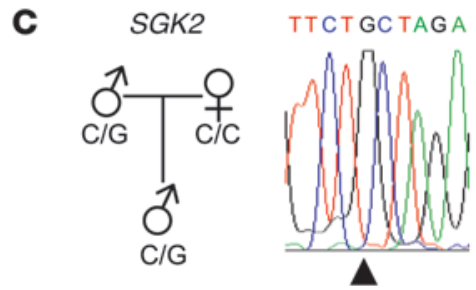

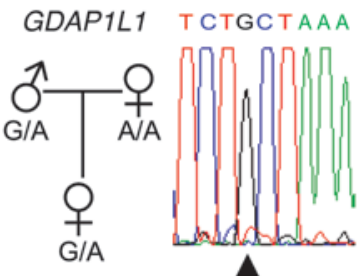

\section{Results}

The $20 q$ common deleted region contains a cluster of genes that exhibit primate-restricted imprinting. Having previously shown that one of the genes in the $20 \mathrm{q}$ common deleted region (CDR), L3MBTL1, is imprinted (24), we considered the possibility that other genes in the vicinity may also be imprinted and contribute to the pathogenesis of malignancies associated with $20 \mathrm{q}$ deletions. We therefore determined allele-specific expression of neighboring genes in the 20q CDR (Figure 1A). Most genes (SFRS6, IFT52, MYBB, JPH2, and C20orf111) were biallelically expressed in normal peripheral blood cells, but SGK2 (encoding a serine/threonine kinase) and GDAP1L1 (encoding a protein of unknown function) each exhibited monoal- lelic expression in 2 different hematopoietic cell types (Figure 1B and Supplemental Table 1; supplemental material available online with this article; doi:10.1172/JCI66113DS1). Studies of 6 families showed that, like L3MBTL1, both SGK2 $(n=4)$ and GDAP1L1 $(n=2)$ were expressed from the paternal allele (Figure 1C and Supplemental Figure 1).

To identify candidate imprinting control regions, $\mathrm{CpG}$ islands and islets within the myeloid CDR (Figure 1A) were identified in silico and confirmed by immunoprecipitation of methylated DNA from normal individuals using an anti-methylcytosine antibody. This approach identified 4 methylated regions in the vicinity of L3MBTL1 and SGK2 and 1 in the GDAP1L1 
A

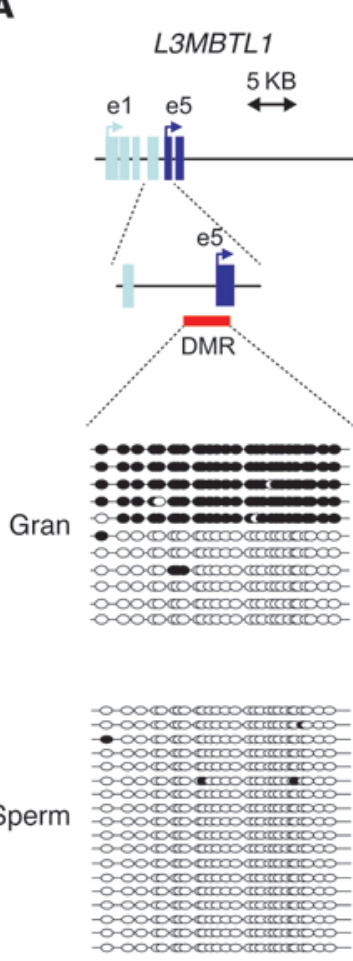

Human L3MBTL1/SGK2 locus
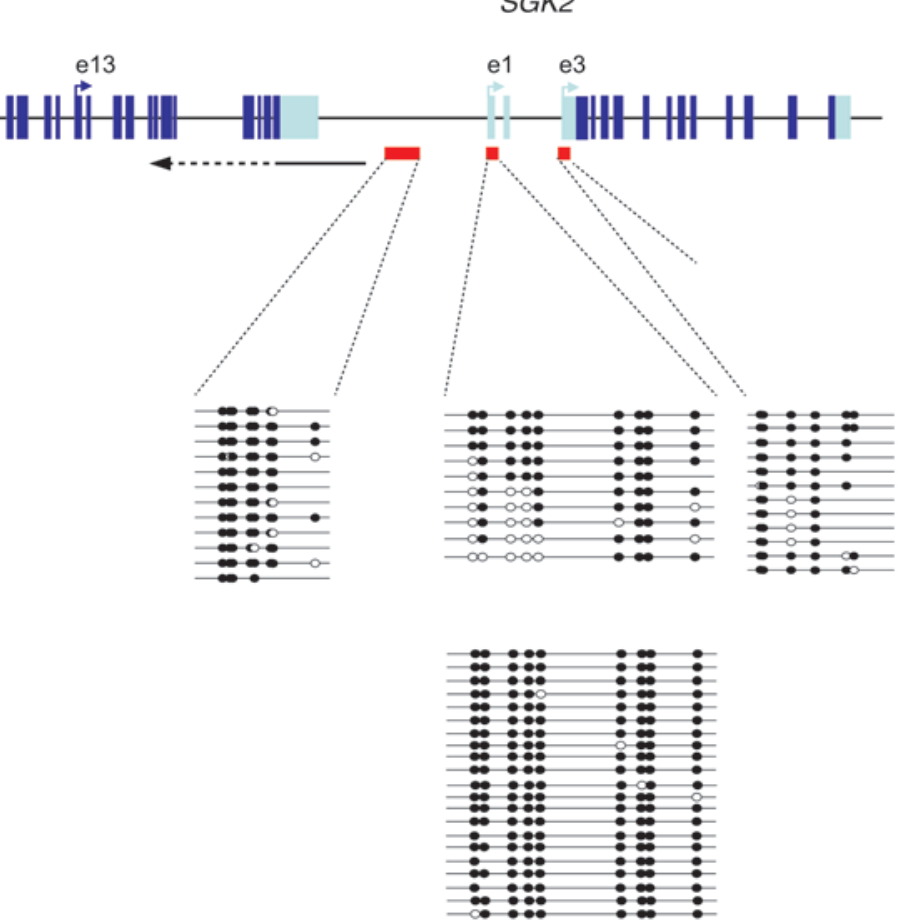

Figure 2

Characterization of the $\angle 3 M B$ TL1 germline DMR in primates. (A) L3MBTL1 and SGK2 locus, indicating the positions of the methylated regions identified by in silico analysis (red bars) and methylated DNA immunoprecipitation from normal human sperm and granulocytes. The DMR in L3MBTL1 promoter 2 is indicated. Each line represents a unique bisulphite-treated PCR product. Methylated and unmethylated $\mathrm{CpGs}$ are represented by filled and unfilled circles, respectively. (B) L3MBTL1 promoter 2 is a germline DMR in primates. Sequence analysis of bisulphite-treated DNA from macaque cells. Methylated and unmethylated $\mathrm{CpG}$ s are represented by filled and open circles, respectively. $\mathrm{M} 1$ and $\mathrm{M} 2$, males 1 and 2; GV, germinal vesicle; MII, meiosis II. The allele for a $\mathrm{C} / \mathrm{T}$ SNP is indicated for the muscle data.
B

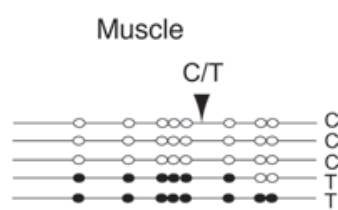

Sperm (M1)

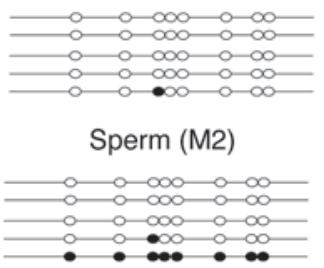

Oocytes

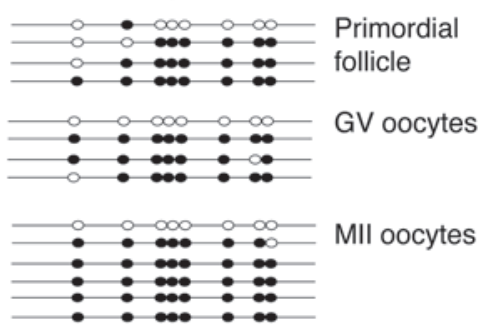

Macaque L3MBTL1 DMR 
A

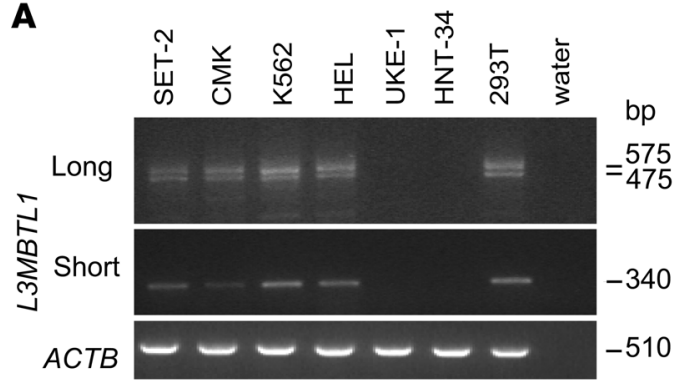

B

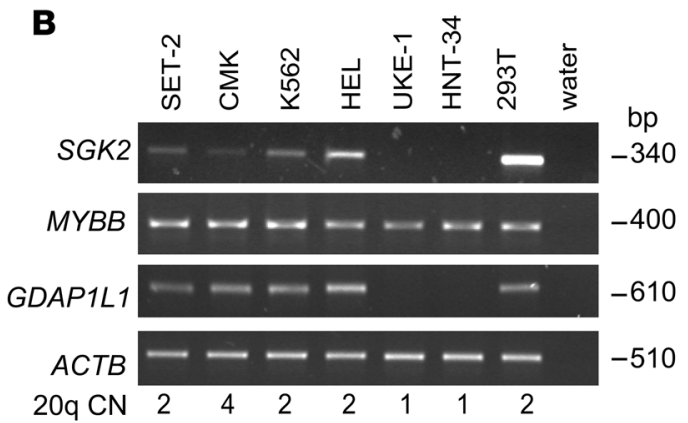

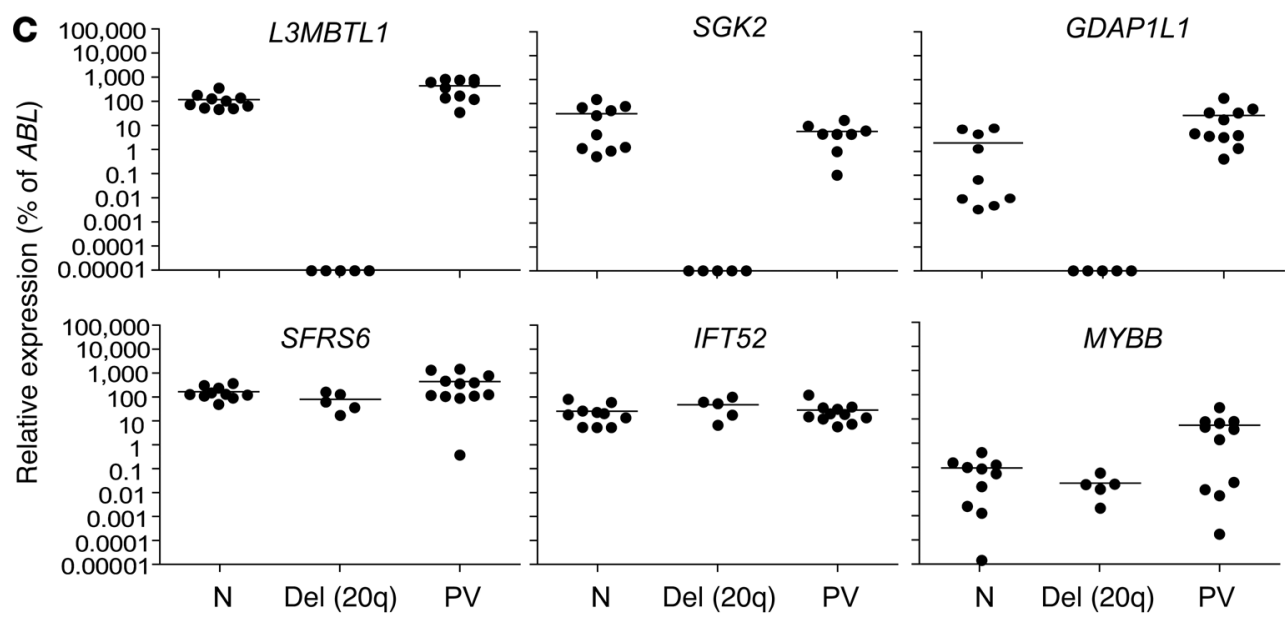

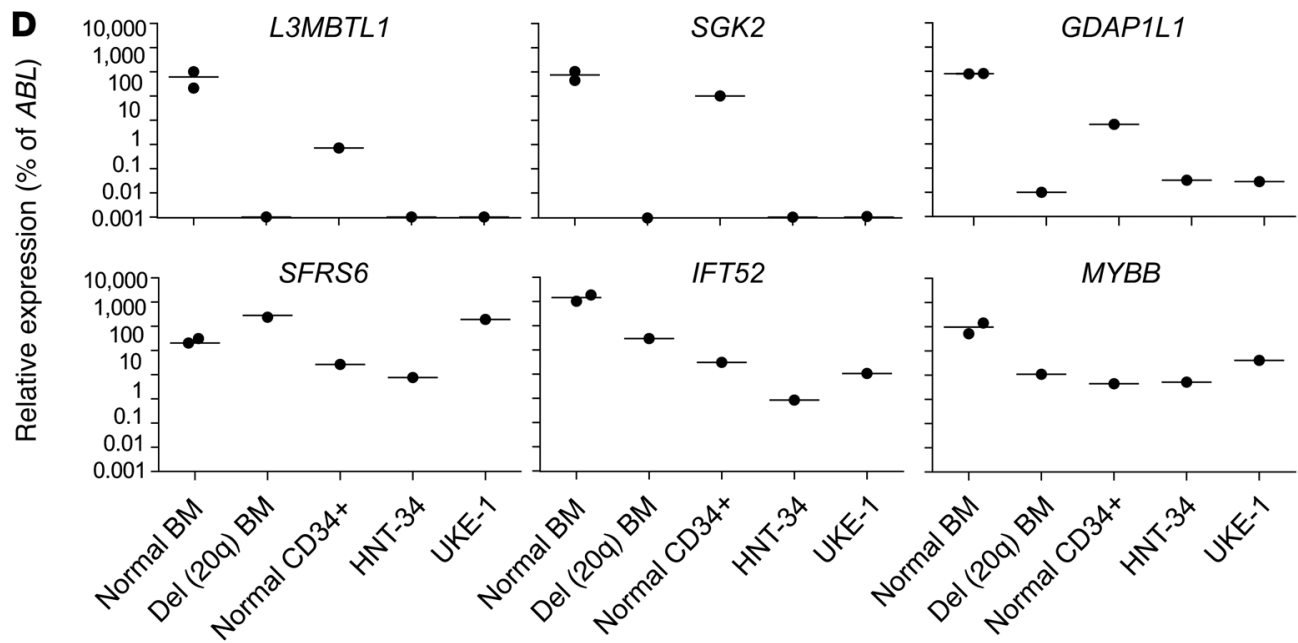

Figure 3

Expression of imprinted genes is lost in hematopoietic cells carrying the 20q deletion. (A) RT-PCR analysis of the full-length (exon 9-14) and short (exons 13a-14) isoforms of L3MBTL1 using cell line cDNAs as indicated. (B) RT-PCR analysis of SGK2, GDAP1L1, MYBB, and $A C T B$ using cell line cDNAs as in A. Copy number of the $20 q$ region (CN) was determined by FISH. (C) qPCR analysis of granulocyte cDNAs in normal (N) patients, patients with 20q deletion [Del(20q)], and PV patients without 20q deletion. (D) qPCR analysis of BM from normal patients and from patients with $20 \mathrm{q}$ deletion, purified CD $34^{+}$cells, and $20 \mathrm{q}$ deletion cell lines. In $\mathbf{C}$ and $\mathbf{D}$, primers for qPCR of L3MBTL1 were in exon 10 and exon 11.

Loss of expression of imprinted genes in neoplastic myeloid cells harboring a 20 q deletion. The imprinted gene cluster is located within the region of $20 \mathrm{q}$ deleted in myeloid malignancies $(11-13,23)$, but levels of L3MBTL1 transcripts were reported to be similar in cells from patients with and without a 20q deletion (13). However, interpre- tation of these results is complicated by several issues: (a) the fact that the presence of even a small fraction of nondeleted cells in patient samples would mask loss of expression in those carrying the $20 \mathrm{q}$ deletion; (b) the presence of an antisense transcript, which was detectable by the primers used by Bench et al. (13) and extend- 
ed into the 3 ' exons of L3MBTL1 (Supplemental Figure 3, A and B); (c) the demonstration that the L3MBTL1 locus was transcriptionally complex with truncated and alternatively spliced transcripts (Supplemental Figure 3A).

In order to circumvent the issue of contaminating nondeleted cells, we studied 2 hematopoietic cell lines (UKE-1 and HNT-34) that carry a cytogenetically detectable $20 \mathrm{q}$ deletion, the presence of which was confirmed by FISH (data not shown). Using $5^{\prime}$ primers that do not detect the antisense transcript, we found that L3MBTL1 transcripts were undetectable in both $20 \mathrm{q}$ deleted cell lines, but were readily apparent in 4 other myeloid cell lines (Figure 3A). Strand-specific RT-PCR showed that both the sense and the antisense transcripts were expressed from the same parental allele (Supplemental Figure 3C), which indicates that the antisense transcript does not function in cis to silence L3MBTL1 expression. The antisense transcript was detectable in HNT-34 cells using nonquantitative RT-PCR (Supplemental Figure 3B). However, quantitative RT-PCR (qPCR) demonstrated that, compared with normal cells, the level of the antisense transcript was $\sim 10$ fold lower in cells carrying a 20q deletion (Supplemental Figure 3D), likely representing very-low-level transcription from the repressed allele, as previously reported for other imprinted loci $(34,35)$. This very-low-level transcription could account, at least in part, for the apparent expression of $L 3 M B T L 1$ in $20 \mathrm{q}$ deletion patients reported previously (13). All 3 L3MBTL1 transcripts were lost in the 2 cell lines carrying a $20 \mathrm{q}$ deletion (Figure 3A), which demonstrated that differential imprinting of individual transcripts did not occur, at least in these cells. Moreover, SGK2 and GDAP1L1 transcripts were also undetectable (Figure 3B). Together, these results raised the possibility that loss of expression of 1 or more of the 3 imprinted genes (L3MBTL1, SGK2, and GDAP1L1) contributes to the pathogenesis of hematological malignancies associated with $20 \mathrm{q}$ deletions.

Expression of genes located within the $20 \mathrm{q}$ CDR was then assessed in peripheral blood granulocytes or BM from patients with a myeloid malignancy carrying a 20q deletion, from cytogenetically normal patients with polycythemia vera (PV), and from hematologically normal individuals. Since even a small number of contaminating nondeleted cells would mask loss of expression, microsatellite PCR was used to quantify the proportion of cells carrying a 20q deletion (Supplemental Figure 3E), and subsequent analysis was restricted to 6 patients in whom the $20 \mathrm{q}$ deletion was present in greater than $98 \%$ of cells (patients 1-6; Supplemental Table 2). qPCR demonstrated that transcripts for L3MBTL1, SGK2, and GDAP1L1 were extremely low or undetectable in peripheral blood granulocytes or BM from all 6 patients with a $20 \mathrm{q}$ deletion, but were readily detectable in all normal individuals and cytogenetically normal PV patients (Figure 3, C and D). In contrast, transcript levels of nonimprinted genes located in the 20q CDR (SFRS6, IFT52, and MYBB) were readily evident in 20q deletion patient samples and cell lines (Figure 3, C and D). A prior report that SGK2 and JPH2 were not expressed in normal BM or BMderived $\mathrm{CD}_{3} 4^{+}$cells used a less sensitive RT-PCR (13). All qPCR data were normalized to $A B L$, which has previously been shown to be the most consistently expressed control gene in hematopoietic cells (36). Since loss of expression might arise through somatic mutation, we sequenced all known promoters, exons, and splice sites for L3MBTL1, SGK2, and GDAP1L1. Several SNPs were identified (Supplemental Table 3), but no somatic mutations were detected, in samples from 23 patients with a 20q deletion (15 MPN and 8 MDS) or from 19 cytogenetically normal PV patients.
In order to extend the dataset further, clonal analysis of individual hematopoietic colonies was performed using samples from an additional 8 patients in whom the deletion was present in a smaller percentage of cells (<95\%; patients 7-14; Supplemental Table 2, Figure 4, A-D, and Supplemental Figure 4, B-H). Colonies were grown from $\mathrm{CD} 34^{+}$cells to minimize contamination by background nondeleted cells, and each colony was assessed for the presence of the $20 \mathrm{q}$ deletion using several informative markers to ensure accurate genotyping and circumvent apparent loss of heterozygosity $(\mathrm{LOH})$ resulting from preferential amplification of smaller alleles (Supplemental Figure 4A). All colonies (GM-CFUs and erythroid burst-forming units [E-BFUs]) carrying the 20q deletion expressed normal levels of MYBB, C20orf111, and IFT52 transcripts, but - in marked contrast - lacked detectable levels of $L 3 M B T L 1$ transcripts (Figure 4, A-D, and Supplemental Figure 4, B-H). The variation in expression levels in normal colonies is likely to reflect differences in cellular composition and maturity of individual colonies. Analysis of SGK2 was precluded by low levels of expression in normal colonies, and GDAP1L1 expression was undetectable in some, but not all, colonies carrying the $20 \mathrm{q}$ deletion (Figure 4 and Supplemental Figure 4, B-H). Interestingly, in patient 14, myeloid colonies expressed GDAP1L1, but erythroid colonies did not (Supplemental Figure 4, G and $\mathrm{H}$ ), which suggests that control of GDAP1L1 imprinting on the retained allele is subject to tighter regulation in the erythroid lineage than the myeloid lineage. The variable loss of GDAP1L1 expression may reflect the fact that GDAP1L1 is located greater than $800 \mathrm{~kb}$ from the germline DMR identified above; relaxed control of monoallelic expression by an imprinting control region has previously been reported for genes at this distance from the controlling element $(37,38)$. Alternatively, the GDAP1L1 locus may be subject to a distinct imprinting mechanism from that regulating the L3MBTL1 and SGK2 genes.

In total, we showed loss of L3MBTL1 expression in all 16 samples (14 patients and 2 cell lines) from individuals with a 20q deletion, which suggests that the paternal allele is consistently lost. MPN patients are elderly, and so parental samples are not available to confirm this; however, it is possible to infer the parental origin of the retained $L 3 M B T L 1$ allele by assessing the methylation status of its DMR. Of the 6 patients studied here, in whom the $20 \mathrm{q}$ deletion was present in the majority of granulocytes and L3MBTL1 expression was lost, the retained L3MBTL1 allele was previously shown to be methylated in 5 patients, but unmethylated in 1 (24). These results were confirmed using quantitative pyrosequencing (39) for all 5 patients for whom samples were available, including the one with an unmethylated retained allele (patient 5; Figure 4E). The discrepancy between expression and methylation in patient 5 could reflect retention of the maternal allele with aberrant hypomethylation, or retention of the paternal allele with additional lesions causing loss of expression. To distinguish between these possibilities, we investigated the methylation status of a second imprinted gene outside the L3MBTL1 cluster, neuronatin (NNAT, ref. 40). NNAT is known to be expressed from its paternal allele, methylated at the maternally inherited promoter, and located within the boundaries of most $20 \mathrm{q}$ deletions. Patient samples lacking a $20 \mathrm{q}$ deletion showed that, as expected, approximately $50 \%$ of $\mathrm{CpG}$ dinucleotides were methylated. In contrast, all patient samples with a $20 \mathrm{q}$ deletion (including patient 5) had hypermethylation at the NNAT DMR (Figure 4E). These data indicate that the maternally derived chromosome 20 was retained in all patients studied and that in patient 5, the retained L3MBTL1 

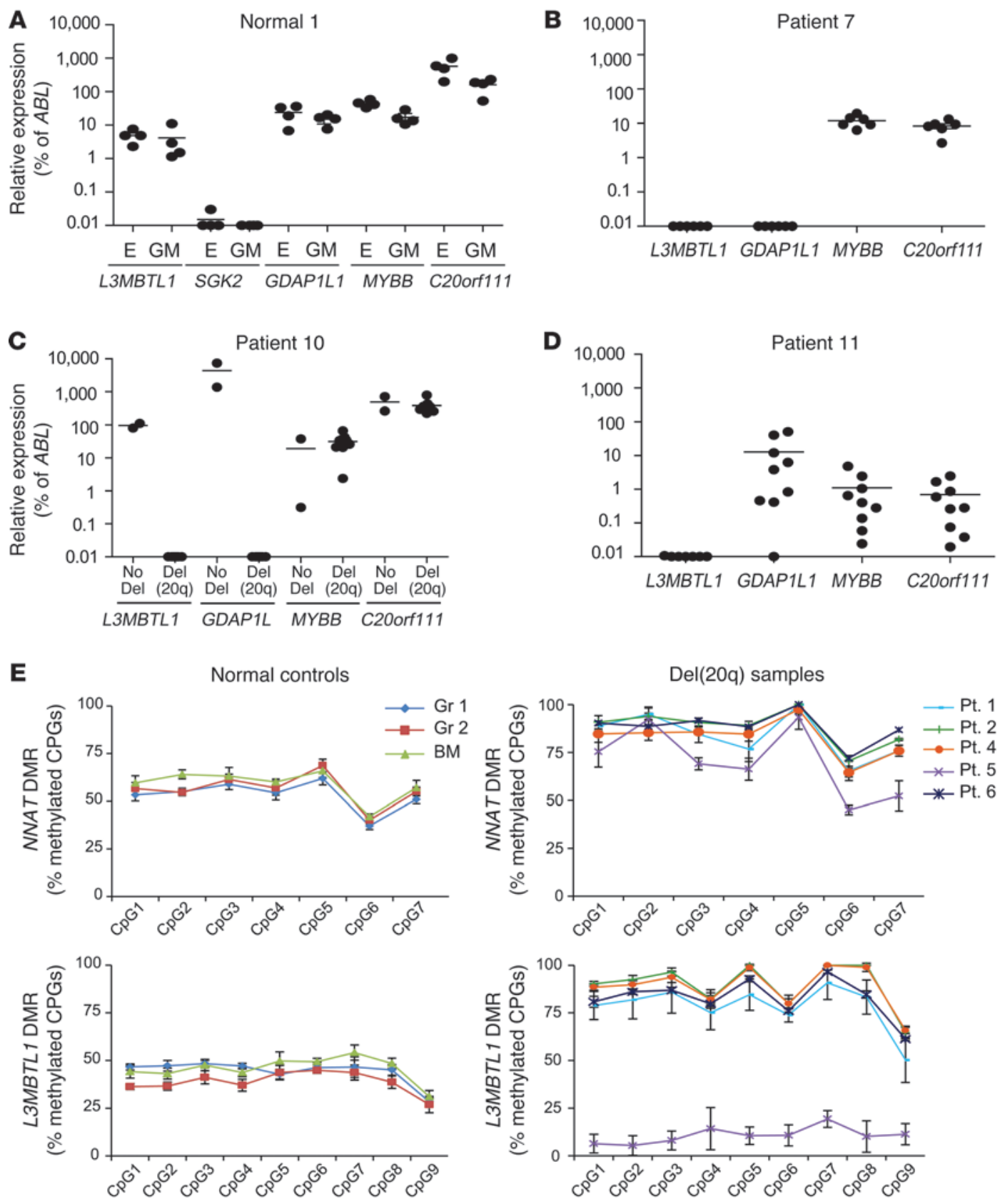

Figure 4

Loss of expression of imprinted genes in hematopoietic colonies carrying a chromosome $20 \mathrm{q}$ deletion. (A-D) Expression of imprinted genes in E-BFUs (E) and GM-CFUs (GM) from a normal individual (A) and in hematopoietic colonies from patients 7, 10, and 11 (B-D; Supplemental Table 2). Each data point represents the mean of 2 qPCR technical replicates. (B) For patient 7, GM-CFUs were analyzed, and all colonies carried the $20 \mathrm{q}$ deletion. (C) For patient 10, E-BFUs were analyzed with or without the $20 \mathrm{q}$ deletion. (D) For patient 11, E-BFUs were analyzed, and all colonies carried the 20q deletion. See Supplemental Figure 4 for further patient sample analyses. (E) Quantitative pyrosequencing of NNAT DMR (chr20:36148604-36150136) and L3MBTL1 DMR (chr20:42143211-42143591) in normal granulocyte bisulphite-treated DNA (Gr1 and Gr2), BM from an MDS patient with cytogenetically normal 20q bisulphite-treated DNA (BM), and the indicated patients with 20q deletion. Each data point represents percent methylation detected at a single $\mathrm{CpG}$ dinucleotide (CpG1-CpG9 on $x$ axis); each pyrosequencing PCR was performed in triplicate.

allele was aberrantly hypomethylated. Taken together, our data demonstrated that myeloid malignancies carrying a $20 \mathrm{q}$ deletion harbor markedly reduced levels of L3MBTL1, SGK2, and/or GDAP1L1 transcripts and indicate that loss of the active paternally inherited locus likely provides a selective advantage.
L3MBTL1 and SGK2 cooperate to regulate hematopoiesis: coordinated silencing maintains megakaryopoiesis and enhances erythropoiesis, but does not provide a selective advantage to stem and early progenitor cells. Hypercellular BM with increased erythropoiesis and aberrant megakaryopoieis are features shared by many MDS and MPN patients. 
A

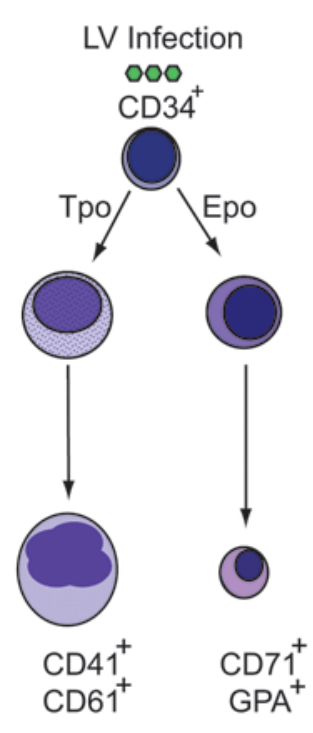

B

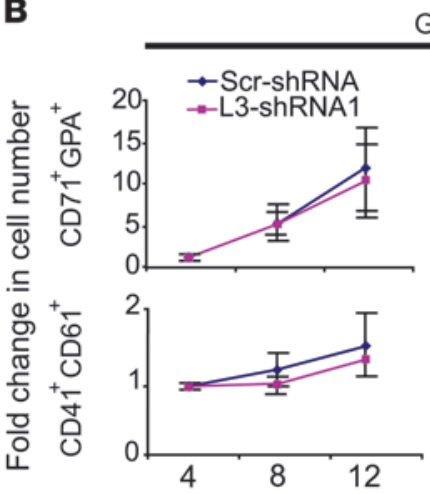

GFP $^{+}$
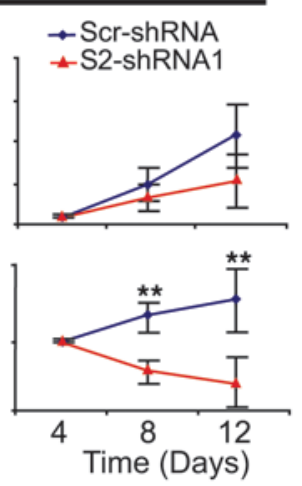

C

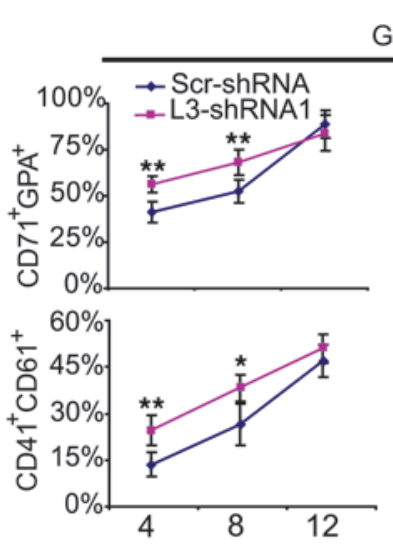

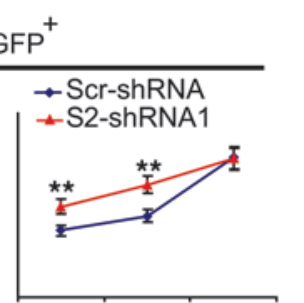

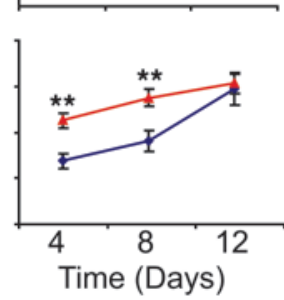

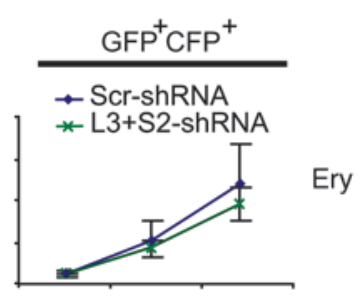
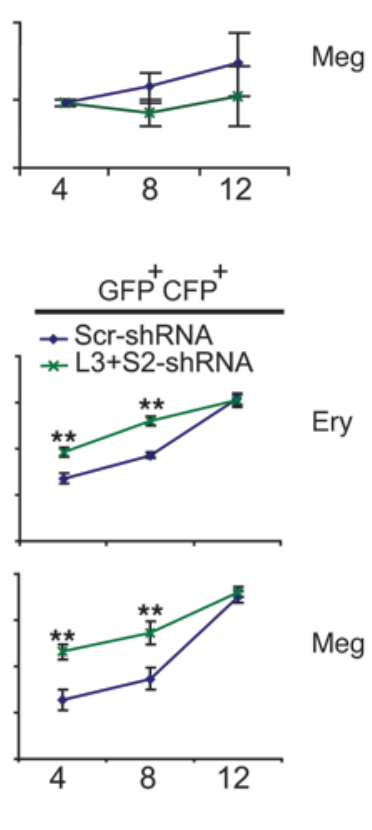

ry

Ery

Meg

\section{Figure 5}

Silencing of $L 3 M B T L 1$ and SGK2 promotes erythroid and megakaryocytic differentiation. (A) Single-phase erythroid (Ery; CD71+GPA+) or megakaryocytic (Meg; $\mathrm{CD} 41^{+} \mathrm{CD} 61^{+}$) culture of $\mathrm{CD} 34^{+}$cells. LV, lentiviral. (B) In vitro cell counts showing the effect on erythroid or megakaryocytic differentiation upon lentiviral shRNA-mediated knockdown of L3MBTL1 (L3-shRNA1), SGK2 (S2-shRNA1), L3MBTL1 and SGK2 together (L3+S2-shRNA), or scrambled control (Scr-shRNA). GFP ${ }^{+}$, single shRNA-LV; GFP+CFP+, double shRNA-LV. (C) Erythroid and megakaryocytic cells, expressed as a percentage of total GFP ${ }^{+}$or GFP ${ }^{+} \mathrm{CFP}^{+}$cells. Error bars indicate SEM of 6 independent experiments. ${ }^{\star} P<0.05$, ${ }^{* \star} P<0.01$ versus scrambled control.

To investigate a pathogenetic role for the imprinted cluster on $20 \mathrm{q}$, we examined the function of L3MBTL1 and SGK2 in the erythroid and megakaryocytic lineages. Transcript levels of both genes were modulated during erythroid and megakaryocytic differentiation of normal CD34+ progenitors and were repressed by 2 independent lentiviral shRNAs for each gene (Supplemental Figure 5, A and B). Inhibition of L3MBTL1 during the differentiation of CD34 $4^{+}$cells did not alter the generation of erythroid $\left(\mathrm{CD} 71^{+} \mathrm{GPA}^{+}\right)$or megakaryocytic $\left(\mathrm{CD} 41^{+} \mathrm{CD} 61^{+}\right)$cells, whereas $S G K 2$ inhibition significantly reduced megakaryocytic cell formation (Figure 5, A and B). Combined knockdown of both L3MBTL1 and SGK2 reversed the inhibitory effect of knocking down SGK2 alone (Figure 5B), which indicates that loss of L3MBTL1 is able to compensate for loss of SGK2 and maintain megakaryopoiesis.

In this monophasic culture system, we noted that the percentage of $\mathrm{GFP}^{+}$cells expressing erythroid or megakaryocytic markers was already increased at day 4 after introduction of shRNAs to L3MBTL1 or SGK2 (Figure 5C and Supplemental Figure 5, C-E), suggestive of an early effect during differentiation. To investigate this possibility, we used a biphasic culture (Figure 6A and Supplemental Figure 6A), in the first phase of which $\mathrm{CD} 71^{+} \mathrm{GPA}^{+/-}$early erythroid cells are generated, but their subsequent differentiation is blocked. In the second phase, the culture conditions are altered to allow differentiation into $\mathrm{CD} 71^{-} \mathrm{GPA}^{+}$late erythroid cells. Late erythroid differentiation was not affected by repressing L3MBTL1 and/or SGK2 (Figure 6B). In contrast, repression of either $L 3 M B$ $T L 1$ or $S G K 2$ alone significantly increased the number of CD71+ early erythroblasts and the percentage of these cells in S phase, without any alteration in apoptosis (Figure 6B and Supplemental Figure 6, B and C). Inhibition of both genes resulted in an even more marked increase in the number of early erythroblasts and in the percentage of cells in $\mathrm{S}$ phase (Figure 6B and Supplemental Figure 6B), which demonstrated a cooperative effect on the proliferation of early erythroid progenitors.

These results predicted that expression of L3MBTL1 and/or $S G K 2$ in cells carrying a $20 \mathrm{q}$ deletion would inhibit erythroid differentiation. To investigate this possibility, L3MBTL1 and/ or SGK2 was expressed in HNT-34 cells (which carry a 20q deletion) derived from a patient with MDS (Supplemental Figure 6D). Hemin-induced expression of GPA, $\alpha$-globin, and $\beta$-globin was suppressed by introduction of L3MBTL1 or SGK2, with expression of both producing significantly more repression of GPA (Figure 6C and Supplemental Figure 6, E and F). In addition, when HNT34 cells were cultured with the demethylating agent 5-azacytidine, 
A

Phase I

IGF1, SCF, Low Epo,

Dexamethasone

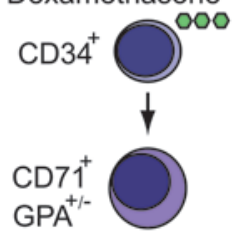

Phase II

Insulin, High Epo,

Transferrin

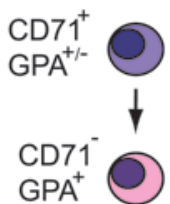

C
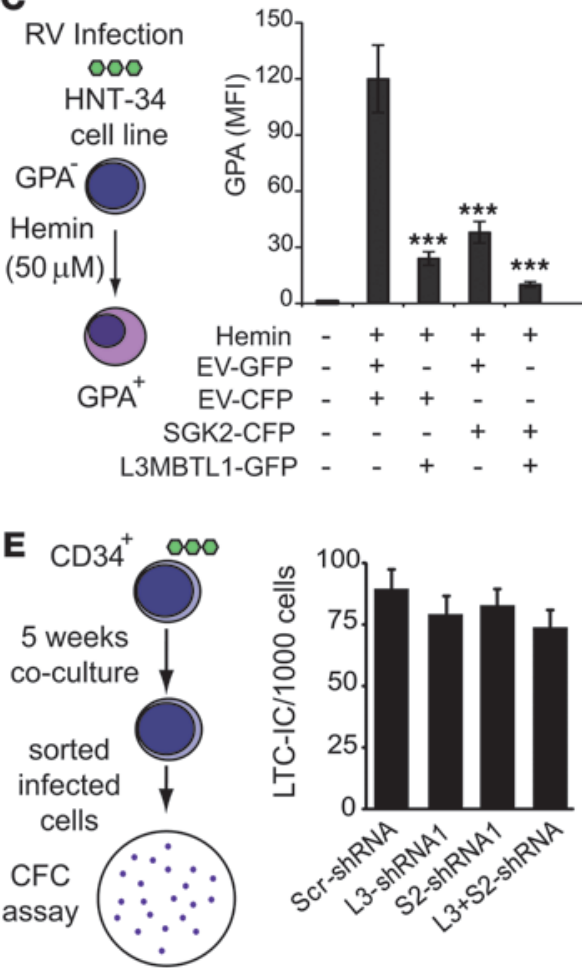

B

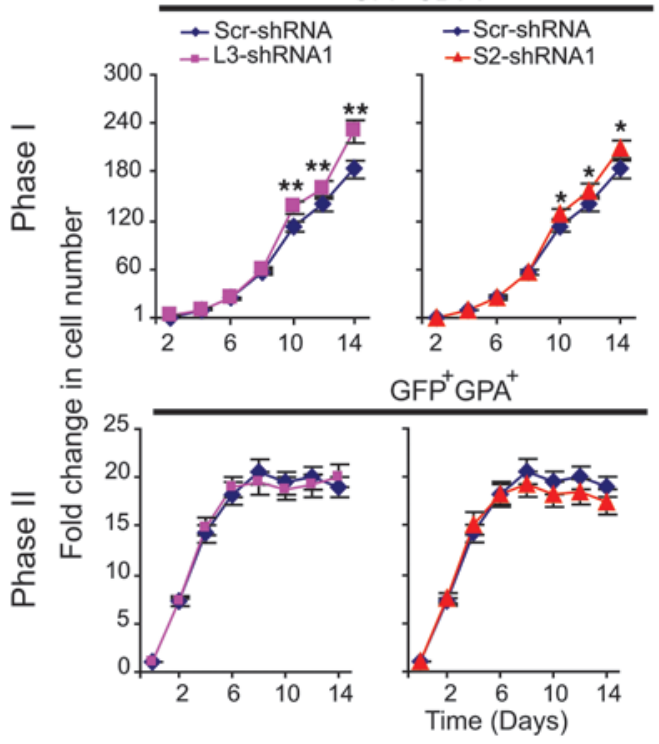

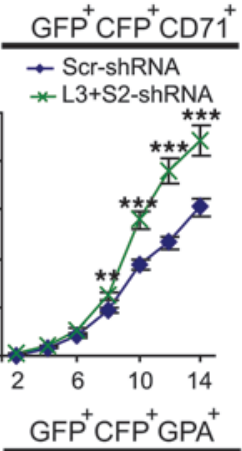

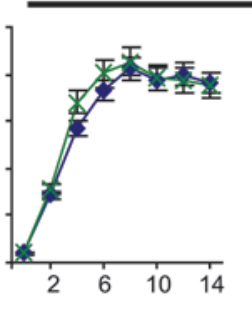

D
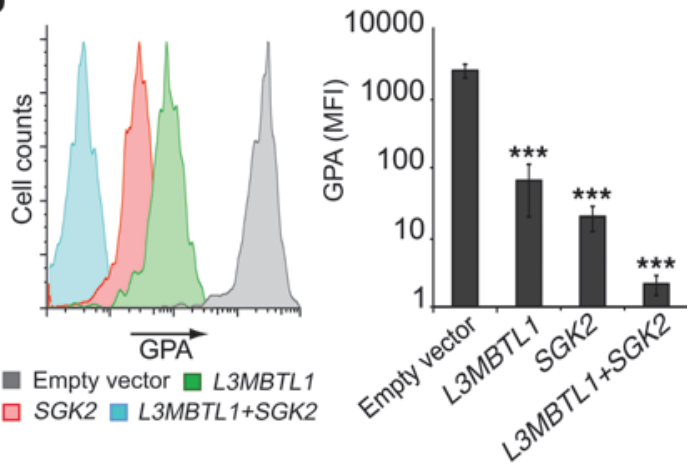

F

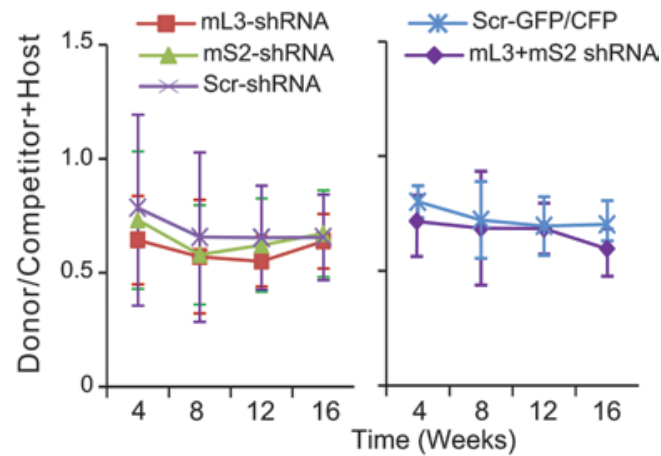

Figure 6

Coordinated silencing of $\angle 3 M B T L 1$ and SGK2 increases proliferation of early erythroid progenitors. (A) Biphasic erythroid culture of CD34+ cells. (B) In vitro cell count after shRNA-mediated knockdown of L3MBTL1, SGK2, L3MBTL1 and SGK2 together, or scrambled control. Error bars indicate SEM of 5 independent experiments. ${ }^{*} P<0.05,{ }^{* \star} P<0.01,{ }^{* \star *} P<0.001$ versus scrambled control. (C) Hemin-induced erythroid differentiation of HNT-34 cells after retroviral (RV) infection. FACS analysis of hemin-induced GPA expression is also shown, summarized as MFI of GPA in 3 independent experiments. EV, empty vector. Error bars indicate SEM. ${ }^{* * *} P<0.001$ versus empty vector. (D) Erythroid differentiation of 20q deletion clones obtained from patient 13. Single-cell clones were grown in erythroid conditions from CD34+ cells retrovirally infected with L3MBTL1, SGK2, L3MBTL1 and SGK2 together, or empty vector. Shown are representative 20q deletion clones and MFI of GPA expression in 20q deletion clones ( $n=39$ [empty vector]; 37 [L3MBTL1]; 27 [SGK2]; 21 [L3MBTL1 and SGK2 together]). ${ }^{* * *} P<0.001$ versus empty vector. (E) LTC-IC assay of lentivirus infected cord blood CD34+ cells. CFC, colony-forming cell. The number of colony-forming cells after 5 weeks is also shown. Error bars indicate SEM of 3 independent experiments. (F) Competitive reconstituted mice were analyzed for 16 weeks for peripheral blood mononuclear cell donor/competitor ratios. CD45.2+ donors - with shRNA-mediated knockdown of murine (m-) L3mbtl1, Sgk2, L3mbtl1 and Sgk2 together, or scrambled control - were competed with equal numbers of CD45.1+CD45.2+ F1 competitors $(n=5)$ and injected into CD45.1+ hosts. 
it was possible not only to induce expression of L3MBTL1 and $S G K 2$, but also to recapitulate the reduced differentiation phenotype expected when the genes are expressed (Supplemental Figure $6, \mathrm{G}$ and $\mathrm{H})$. To extend these results to primary cells, CD $34^{+}$cells from a patient with essential thrombocythemia and a $20 \mathrm{q}$ deletion (patient 13; Supplemental Table 2) were infected with retroviruses expressing L3MBTL1, SGK2, or both and grown as single-cell clones in conditions supporting erythroid differentiation. After 8 days, the presence of a $20 \mathrm{q}$ deletion was confirmed using microsatellite PCR, and GPA expression was assessed by FACS. As shown in Figure $6 \mathrm{D}$, rescue of either $L 3 M B T L 1$ or SGK2 expression resulted in a significant reduction in GPA levels, which were further reduced by combined expression of L3MBTL1 and SGK2. Together, these data were consistent with our knockdown results and demonstrated that L3MBTL1 and SGK2 cooperate to reverse the erythroid phenotype associated with $20 \mathrm{q}$ deletions.

Taken together, these data showed not only that L3MBTL1 and SGK2 exhibit substantial interactions, but also that these interactions vary in different lineages and in successive stages of differentiation. In late megakaryocytic differentiation, loss of L3MBTL1 reverses the inhibitory effects of SGK2 loss, a dominant epistatic (or antagonistic) interaction. In contrast, the combined loss of L3MBTL1 and SGK2 has an additive positive effect on the proliferation of early erythroid progenitors, an observation consistent with the BM erythroid hyperplasia associated with both MDS and MPN.

We considered the possibility that loss of L3MBTL1 and SGK2 function may also provide a selective advantage at the level of HSCs or early progenitor cells. 3 different approaches were pursued. First, lentiviral shRNA constructs were used to repress L3MBTL1 and/or SGK2 in human CD34+ progenitors, which were then assessed in long-term culture initiating cell (LTC-IC) assays. Inhibition of L3MBTL1 and/or SGK2 did not result in altered numbers of E-BFUs, GM-CFUs, granulocyte-erythrocytemonocyte-macrophage CFUs (GEMM-CFUs), or total colonies at the end of the LTC-IC culture period (Figure 6E and Supplemental Figure 6G). Second, lentiviral shRNA constructs were used to repress $L 3 m b t l 1$ and/or Sgk2 in murine lineage-negative BM cells (Supplemental Figure 6H), which were then assessed in spleen CFU (S-CFU) assays. Inhibition of L $3 \mathrm{mbtl} 1$ and/or Sgk2 did not result in alteration in the number or size of the S-CFUs (Supplemental Figure 6I and data not shown). Third, competitive BM transplantation experiments were performed. Lentivirally infected test BM cells (CD45.2 $2^{+}$were mixed with equal numbers of $\mathrm{CD} 45.2^{+} \mathrm{CD} 45.1^{+}$(F1) competitor BM cells and transplanted into lethally irradiated $\mathrm{CD} 45.1^{+}$recipients. Knockdown of $\mathrm{L} 3 \mathrm{mbtl} 1$ and Sgk2 was sustained in lineage-negative CD $45.2^{+}$BM cells 16 weeks after reconstitution (Supplemental Figure 6J). FACS analysis 16 weeks after transplant showed that inhibition of $L 3 m b t l 1$ and/or Sgk2 did not alter the contribution of CD 45.2+ test cells to peripheral blood (Figure 6F), to BM progenitor cells (Lin-Sca- $1^{+}$ $\mathrm{c}^{-\mathrm{Kit}^{-}}$, common myeloid progenitor, granulocyte-macrophage progenitor, and megakaryocyte-erythroid progenitor; Supplemental Figure $6 \mathrm{~K}$ ), or to more mature cells positive for B220, CD71, Mac-1, or CD3e (Supplemental Figure 6L). Together, these results indicate that inhibition of L3MBTL1 and/or SGK2 does not result in a selective advantage for stem or early progenitor cells and are consistent with previous evidence that several known driver mutations are not associated with an expanded HSC compartment (28, 41-47).
SGK2 and L3MBTL1 cooperate to regulate MYC transcription. To gain insight into the molecular basis for the cooperation between L3MBTL1 and SGK2, we focused on 3 known targets of L3MBTL1: MYC, RUNX1, and CCNE1 $(15,16)$. Transcript levels of all 3 genes were increased by repression of L3MBTL1 in erythroid cells generated by differentiation of cord blood progenitors (Figure 7A). Repression of SGK2 did not alter expression of any of the 3 genes, but combined repression of both L3MBTL1 and $S G K 2$ gave rise to a striking increase in transcript levels of MYC, but not of RUNX1 or CCNE1 (Figure 7A and Supplemental Figure 7A). The synergistic increase in MYC expression was accompanied by similarly increased levels of H3K4me3 and RNA polymerase II at the MYC promoter and transcription start site, respectively (Figure 7B and Supplemental Figure 7, B and C), which indicates that L3MBTL1 and SGK2 cooperate by enhancing $M Y C$ transcription, rather than by altering $M Y C$ transcript stability. L3MBTL1 binding at the MYC promoter was reduced, as expected, by shRNA-mediated repression of L3MBTL1, but was not affected by repression of SGK2 alone, and no additional effect was obtained by repressing both L3MBTL1 and SGK2 (Figure 7C and Supplemental Figure 7C), which indicates that SGK2 does not modulate $M Y C$ transcription by regulating the binding of L3MBTL1 to the MYC promoter.

We therefore searched for other mechanisms whereby SGK2 might influence MYC transcription. Little is known about the biological functions of $S G K 2$, but it has been shown that SGK family members are induced by MAPK signaling (48) and activated by the PI3K pathway (49). A potential link is provided by prior observations that $M Y C$ transcription is transiently upregulated by BRG1 (also known as SMARC4) (50), the ATPase subunit of a mammalian SWI/SNF-like chromatin remodeling complex (51), and that serine phosphorylation of BRG1 prevents its recruitment to DNA (52). We therefore tested the hypothesis that SGK2 might downregulate MYC transcription by inducing phosphorylation and inactivation of BRG1. Consistent with this model, ChIP experiments demonstrated that repression of $L 3 M B T L 1$ did not alter BRG1 binding to the MYC promoter. In contrast, repression of $S G K 2$, either alone or in combination with repression of $L 3 M B T L 1$, was accompanied by a marked increase in BRG1 recruitment (Figure 7D and Supplemental Figure 7E). Restoration of SGK2 expression in HNT-34 cells, which carry a 20q deletion, was accompanied by increased levels of serine-phosphorylated BRG1, reduced binding of BRG1 to the MYC promoter, and caused a concomitant decrease in MYC expression (Figure 7, E-G, and Supplemental Figure 7F). Furthermore, expression of L3MBTL1 and/or SGK2 was rescued in $\mathrm{CD}_{3} 4^{+}$cells a carrying $20 \mathrm{q}$ deletion that were derived from a patient with essential thrombocythemia (patient 13; Supplemental Table 2 and Figure 7H). Analyses of single-cell clones demonstrated that the $20 \mathrm{q}$ deletion clone generally expressed higher levels of MYC than WT clones, an effect that was partially reversed by expression of $L 3 M B T L 1$ or $S G K 2$ alone and completely reversed by their combined expression (Figure $7 \mathrm{H}$ ).

Together, these results indicate that coordinate loss of expression of L3MBTL1 and SGK2, as a consequence of a 20q deletion, results in cooperative effects on distinct aspects of chromatin structure (Figure 7I). L3MBTL1 is known to regulate nucleosomal compaction, and we showed here that SGK2 inactivated BRG1, a key ATP-dependent helicase within the SWI/SNF complex that regulates nucleosomal positioning. 

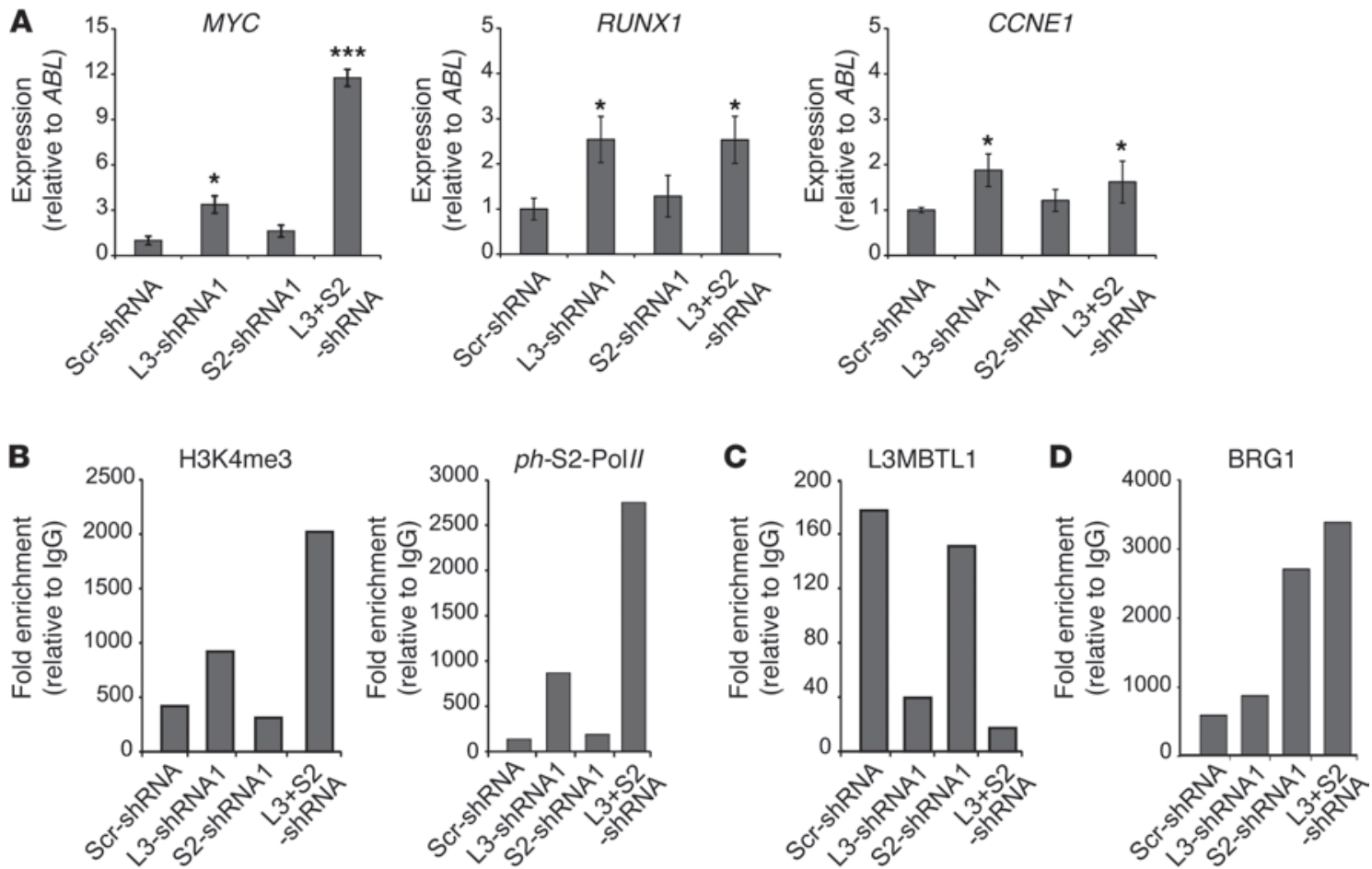

E
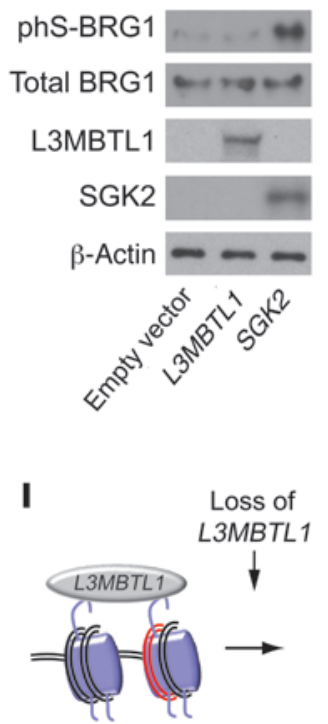

F
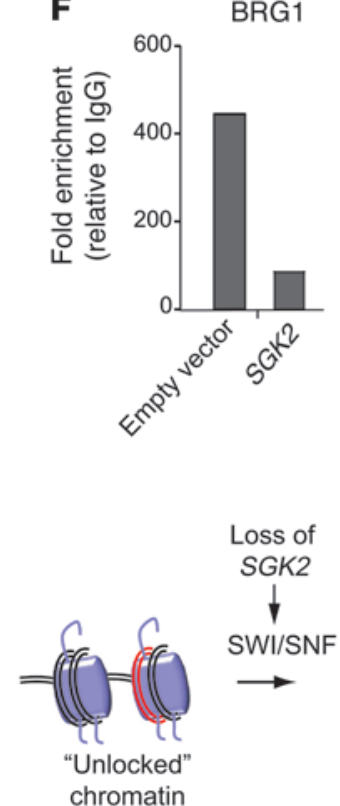
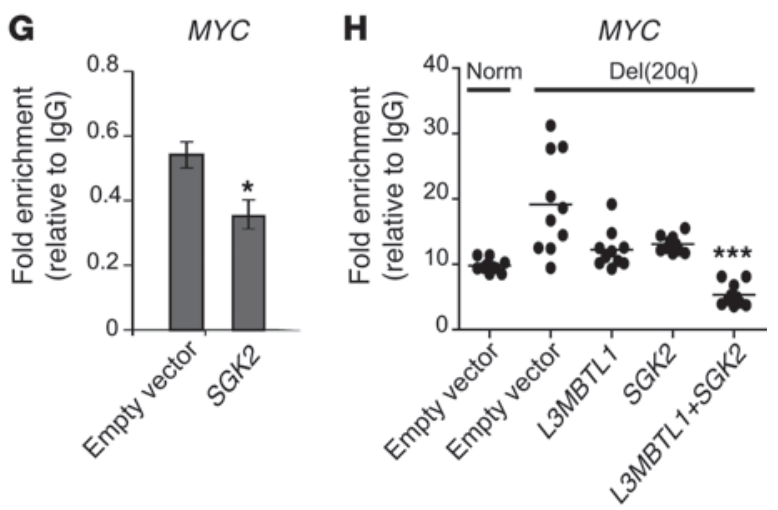

Figure 7

Coordinated silencing of $L 3 M B T L 1$ and SGK2 increases MYC transcription. (A) qPCR analysis of MYC, RUNX1, and CCNE1 transcript levels in cord blood-derived erythroid cells after lentiviral knockdown of L3MBTL1, SGK2, L3MBTL1 and SGK2 together, or scrambled shRNA control $(n=6) .{ }^{*} P<0.05,{ }^{* *} P<0.001$ versus scrambled control. (B-D) ChIP PCR analysis for binding of the indicated proteins at the MYC promoter (H3K4me3, L3MBTL1, and BRG1) or transcription start site (ph-S2-Poll/) in erythroid cells infected with lentiviral shRNA as in A. Representative experiments are shown. Similar results were obtained in 2 further independent experiments (Supplemental Figure 7, B-E). Mean of duplicate qPCR measurements are shown. (E) Western blot from HNT-34 cells after immunoprecipitation with antibody against BRG1. Cells were transfected with retroviral expression constructs as indicated. (F and G) HNT-34 cells infected with retrovirus expressing SGK2 or control and analyzed for binding of BRG1 (as described above) to the MYC promoter (F) or MYC transcript levels $(\mathbf{G}) .{ }^{*} P<0.05$ versus control. (H) Expression of $M Y C$ (qPCR) in single-cell clones grown in erythroid conditions. CD34+ cells from patient 13 were infected with retroviruses expressing $L 3 M B T L 1$ and/ or SGK2 as indicated. Each data point represents 1 clone; horizontal lines denote means. $n=10$ in each case. ${ }^{* \star *} P<0.001$ versus empty vector-containing $20 \mathrm{q}$ deletion clones. (I) Collaboration between L3MBTL1 and SGK2. 


\section{Discussion}

Our data provided a pathogenetic link between an acquired cancer-associated genetic lesion and an imprinted gene cluster. Moreover, they revealed a new tumorigenic mechanism associated with acquired chromosome deletions. Some somatic deletions remove the remaining normal allele of a heterozygously inactivated tumor suppressor gene (e.g., TP53, RB, and p16, refs. 53$55)$, whereas others result in haploinsufficiency (e.g., $\alpha$-catenin, RPS14, and miR-145 and miR-146a; refs. 7-9). Here, we identified a third mechanism in which a deletion removes actively expressed alleles of an imprinted gene cluster, resulting in the coordinate and complete loss of expression of several genes. Importantly, the 3 mechanisms are not mutually exclusive, and our data do not exclude an additional pathogenetic role for other genes on $20 \mathrm{q}$. Our results have relevance extending well beyond the specific example of $20 \mathrm{q}$ deletions. Monoallelic or allelically imbalanced transcription can reflect processes other than imprinting (e.g., regulatory element polymorphisms), and it has also been reported that monoallelic expression is much more widespread than previously recognized (56). These considerations raise the possibility that the mechanism described here (marked loss of expression of multiple genes) may also be a feature of other examples of malignancy-associated, acquired, large-scale genetic loss, including other deletions and monosomies as well as regions of uniparental disomy.

We have previously shown that L3MBTL1 is imprinted (24), and here we describe that it is part of a previously unrecognized imprinted gene cluster that arose in the primate line after its divergence from other placental mammals. L3MBTL1, SGK2, and GDAP1L1 were expressed from the paternally derived allele, which suggests a common regulatory mechanism. We also identified a promoter of $L 3 M B T L 1$ as a germline DMR, unmethylated in sperm but methylated in oocytes, and therefore a strong candidate for the imprinting control region. Deletions of chromosome $20 \mathrm{q}$ resulted in markedly reduced expression of L3MBTL1 and SGK2, which indicates that monoallelic expression of L3MBTL1 and SGK2 is tightly regulated, perhaps reflecting the proximity of both to the DMR. In contrast, loss of expression of GDAP1L1, located approximately $800 \mathrm{~kb}$ away from the other 2 genes, was observed in some, but not all, individuals with a $20 \mathrm{q}$ deletion. This is consistent with the idea that the influence of the DMR becomes attenuated over large distances, but our data do not exclude the possibility that GDAP1L1 is independently regulated, perhaps in a lineage-restricted or disease-associated manner. Of particular note, monoallelic expression of all 3 genes was a recent evolutionary development; to our knowledge, this represents the first example of primaterestricted imprinting.

Our data demonstrated that acquired deletions of $20 \mathrm{q}$ were associated with consistent loss of the active alleles of L3MBTL1 and SGK2. For L3MBTL1, this pattern was observed in all 16 samples studied, patients 1-14 (Supplemental Table 2) together with cell lines HNT-34 and UKE-1, and our results therefore indicate a pathogenetic role for loss of the paternally derived active locus. Our results do not reflect bias in the selection of samples for analysis. Although we were able to study a minority of patients with a $20 \mathrm{q}$ deletion, patient selection was determined solely by the technical challenges associated with detecting loss of expression in a clonal subpopulation of deleted cells present in a mixed population containing nondeleted cells. Studies of peripheral blood granulocytes were therefore restricted to the minority of patients in whom at least $98 \%$ of cells carried the deletion; for colony assays, we were limited to those samples from which it was possible to grow adequate numbers of colonies (a particular problem in MDS) carrying an unambiguous deletion. The latter was assessed using multiple microsatellite markers to avoid artifacts that can result from preferential amplification of smaller alleles, especially when starting from small amounts of low-molecular weight DNA. Moreover, we found that individual hematopoietic colonies were often contaminated by background cells (e.g., macrophages and platelets) present in the methylcellulose; to reduce this, we introduced a CD34 selection step prior to seeding cells into methylcellulose.

It is instructive to compare our results with previous studies of L3MBTL1 expression in hematopoietic cells with and without a 20q deletion. MacGrogan and colleagues reported detectable expression of L3MBTL1 in some, but not all, cell lines with $20 \mathrm{q}$ LOH and in 15 AML samples, none of which had a 20q deletion (23). However, LOH in cell lines with complex genomic rearrangements may not reflect the same pathogenetic mechanisms as a simple cytogenetically detectable $20 \mathrm{q}$ deletion in patient samples; for this reason, our mapping of the CDR was restricted to samples from MPN or MDS patients (11-13). It has been reported that L3MBTL1 transcript levels in peripheral blood granulocytes, detected using primers from the $3^{\prime}$ region of the L3MBTL1 locus, were not affected by the presence of a $20 \mathrm{q}$ deletion (13). However, the conclusions of this study were limited by several caveats, including the complex transcriptional architecture of the locus with the possibility that a subset of transcripts might not be imprinted, and the issue of contaminating normal undeleted cells (up to $10 \%$ of cells did not carry a $20 \mathrm{q}$ deletion). This problem was compounded by the fact that normal granulocyte expression levels varied considerably between individuals ( $\sim 6$-fold). In the current study, we demonstrated that multiple different L3MBTL1 transcripts were lost in association with $20 \mathrm{q}$ deletions, which indicates that imprinting affects all known L3MBTL1 transcripts. Here, we took great care to minimize contamination by nondeleted cells in our studies of peripheral blood granulocytes and hematopoietic colonies. Importantly, our data also demonstrated the presence of an antisense transcript overlapping the 3 ' region of the L3MBTL1 locus, with low-level expression from the retained allele potentially masking loss of expression of sense transcripts when using primers from the 3 ' region (13).

Targeted inactivation of SGK2 has not been reported, but mice lacking L3MBTL1 do not exhibit a detectable hematopoietic phenotype (57). This observation is likely to reflect a requirement for loss of $L 3 M B T L 1$ activity to cooperate with additional lesions, such as loss of SGK2 expression. In MPN patients, the absence of microdeletions affecting L3MBTL1 and SGK2 (58) argues that loss of additional genes (on 20q, but outside the CDR) may well be required, a scenario reminiscent of prior data implicating several different genes in the pathogenesis of $5 \mathrm{q}$ deletions associated with MDS (7-9, 59). Moreover, our results do not exclude a further requirement for cooperation between $20 \mathrm{q}$ deletions and lesions elsewhere in the genome.

Since myeloid malignancies associated with $20 \mathrm{q}$ deletions generally occur in elderly individuals, parental samples are not available to ascertain the parental origin of the retained allele. We instead assessed the methylation status of patients with $20 \mathrm{q}$ deletions that lacked L3MBTL1 expression in their granulocytes (Figure 4E and ref. 24). The retained $L 3 M B T L 1$ allele was methylated as expected 
in most patients, but was unmethylated in patient 5. Analysis of NNAT methylation was consistent with retention of the maternal chromosome in this and all other tested patients that harbored 20q deletions. This suggests the retained L3MBTL1 allele has undergone aberrant hypomethylation, a phenomenon well established in multiple malignancies (60). Our results do not exclude the possibility that in a minority of patients, the active paternal allele was retained, and that other acquired genetic or epigenetic mechanisms explain the coordinate loss of expression of L3MBTL1 and SGK2. Such mechanisms may account for the previous report of a patient harboring $220 \mathrm{q}$ deletions in distinct clones and affecting the maternal and paternal chromosomes (61). Alternatively, rare $20 q$ deletions affecting the repressed maternal allele may be associated with different molecular lesions, such as haploinsufficiency for, or mutation of, other target genes on 20q, perhaps in concert with distinct lesions elsewhere in the genome.

Loss of L3MBTL1 and/or SGK2 did not result in an advantage for early stem or progenitor cells, as assessed by LTC-IC, S-CFU, or competitive BM transplantation studies. These results were consistent with previous reports showing that several driver mutations found in human myeloid malignancies - including $B C R-A B L$ $(46,47)$, RUNX1 inactivation (42), NRAS activation (45), FLT3-ITD (43), and $J A K 2^{V 617 F}(28,41,44)$ - are not associated with a selective advantage in the HSC compartment and may even impair the function of early stem and progenitor cells. These observations may be related to oncogene-induced senescence, a phenomenon most studied in solid tumors, but also triggered by leukemogenic fusion proteins (62), and suggest that additional lesions are required for the progeny of a mutated HSC to acquire a clonal advantage.

In chronic myeloid malignancies, the clinical phenotype reflects perturbation of late stages of hematopoiesis. Thus, in the chronic phase of disease, $B C R-A B L$ and $J A K 2^{V 617 F}$ are associated with increased granulopoiesis and erythropoiesis, respectively $(41,44$, $47)$. We showed here that coordinate loss of expression of $L 3 M B$ $T L 1$ and $S G K 2$, as a consequence of a single heterozygous deletion, gave rise to dysregulation of erythropoiesis and megakaryopoiesis. Perna and colleagues previously reported that repression of L3MBTL1 alone increases erythroid differentiation of CD34 ${ }^{+}$cells, although they did not detect altered megakaryocyte differentiation (63), perhaps reflecting differences in the cytokine combination used. Here, we demonstrated that repression of L3MBTL1 and $S G K 2$ cooperated to dysregulate both erythropoiesis and megakaryopoiesis. Furthermore, our data demonstrated that L3MBTL1 and SGK2 interacted in distinct ways, depending on cellular context. In megakaryocytic differentiation, repression of L3MBTL1 compensated for the inhibitory effects of SGK2 loss. In contrast, proliferation of early erythroid progenitors was enhanced cooperatively by repression of both $L 3 M B T L 1$ and SGK2. Our results therefore identified $L 3 M B T L 1$ and $S G K 2$ as potential therapeutic targets; however, future approaches will need to take into account the fact that increasing L3MBTL1 activity alone would be predicted to unmask the detrimental effect of SGK2 loss on megakaryopoiesis.

Finally, we demonstrated that L3MBTL1 and SGK2 collaborate during erythropoiesis in the epigenetic regulation of $M Y C$, but not other known human L3MBTL1 target genes. Repression of L3MBTL1 has been shown to relax nucleosomal compaction and is associated with increased MYC expression in cell lines (16). Here, we showed that $S G K 2$ regulated phosphorylation of BRG1, a critical ATPase subunit of mammalian SWI/SNF-like chromatin remodeling complexes $(51,64)$, and modulated BRG1 recruitment to the MYC promoter. Coordinated repression of both L3MBTL1 and $S G K 2$ resulted in a synergistic increase in $M Y C$ expression as a consequence of cooperation between the distinct chromatin-modifying activities of L3MBTL1 and the SWI/SNF complex. L3MBTL1 recognizes $\mathrm{H} 4 \mathrm{~K} 20 \mathrm{me} 1$ and $\mathrm{H} 1 \mathrm{bK} 26 \mathrm{me} 1 / 2$ and compacts chromatin, either by acting as a molecular bridge between adjacent nucleosomes or by binding to an individual nucleosome and altering internucleosomal interactions (16). In contrast, SWI/SNF complexes regulate gene expression by inducing nucleosomal repositioning that alters accessibility of regulatory DNA to the transcriptional machinery (64). The model in Figure 7I is consistent with the antagonistic effect of polycomb group proteins and SWI/ SNF complexes $(64,65)$ and has parallels with the observation that a lymphoma-associated amplicon contains 2 genes, JAK2 and $J M J D 2 C$, increased expression of which cooperates to promote lymphoma development (66). Moreover, our results are also in accord with the demonstration that downregulation of MYC is essential for eythroid differentiation and that a 2- to 3-fold increase in MYC expression promotes proliferation of early erythroblasts (67). The coordinate regulation of gene dosage by genomic imprinting at closely linked genes functioning in distinct molecular pathways aberrant in hematological malignancy has important evolutionary implications for our understanding of the effect of epigenetic control on disease development. Together, our data revealed previously unrecognized biological links between the epigenetic regulator L3MBTL1 and the little-studied serine/threonine kinase SGK2 and underlined the complex molecular and cellular consequences of "simple" cancer-associated chromosome deletions.

\section{Methods}

Patient samples. See Supplemental Methods.

Hematopoietic colony assays. Peripheral blood mononuclear cells from normal individuals and $\mathrm{CD} 34^{+}$cells from $20 \mathrm{q}$ deletion patients were isolated as described above. Cells were grown at a density of $1-5 \times 10^{4}$ cells $/ \mathrm{ml}$ in H4034 methylcellulose medium (Stem Cell Technologies) for 14-21 days according to the manufacturers' instructions. Individual E-BFUs or GMCFUs were picked by aspiration and directly added to $50 \mu \mathrm{l}$ RLT buffer. DNA and RNA were isolated as described below.

FISH. See Supplemental Methods.

Differentiation of $\mathrm{CD}_{3} 4^{+}$cord blood cells. Cord blood was collected from consenting mothers undergoing elective cesarean section surgery in the Rosie maternity Hospital (Cambridge, United Kingdom). CD34 ${ }^{+}$cells were isolated (see Supplemental Methods), then cultured in serum-free media as described previously (68). Briefly, 200,000 CD34+ cells were cultured in StemSpan (Stem Cell Technologies) and 1\% penicillin/streptomycin plus 1 $\mathrm{U} / \mathrm{ml}$ Epo (NeoRecormon), $25 \mathrm{ng} / \mathrm{ml} \mathrm{SCF}$ (Peprotech), $10 \mu \mathrm{M} / \mathrm{ml}$ 2-merceptoethanol, $0.1 \%$ BSA, $4 \mu \mathrm{g} / \mathrm{ml}$ dexamethasone, and $300 \mu \mathrm{g} / \mathrm{ml}$ holo-transferin (iron saturated) for erythroid differentiation, or $100 \mathrm{ng} / \mathrm{ml} \mathrm{Tpo} \mathrm{(Pep-}$ rotech) and $10 \mathrm{ng} / \mathrm{ml} \mathrm{IL-1 \beta}$ (Peprotech) for megakaryocytic differentiation.

Biphasic erythroid differentiation assay. See Supplemental Methods.

LTC-IC assay. See Supplemental Methods.

Single-cell clonality assay. See Supplemental Methods.

Cell culture of cell lines. See Supplemental Methods.

shRNA generation and viral infection. See Supplemental Methods.

Retrovirus generation and transduction. See Supplemental Methods.

Hemin-induced erythroid differentiation assay. HNT-34 cells were infected with retroviruses expressing L3MBTL1-GFP, SGK2-CFP, or both constructs together or empty vector using the following method. Viral supernatant was diluted with fresh RPMI supplemented with $10 \%$ heat-inactivated FCS and 1\% penicillin/streptomycin and used for infecting cells in 
6-well fibronectin-coated plates. 2 rounds of spin infections were carried out within 12 hours using $1,139 \mathrm{~g}$ at $32^{\circ} \mathrm{C}$. Medium was changed after 12 hours of infection, and cells were induced with $50 \mu \mathrm{M}$ hemin in RMPI supplemented with $10 \%$ heat-inactivated FCS and $1 \%$ penicillin/streptomycin. Cell counts and FACS analysis for surface GPA was performed every 24 hours starting 48 hours after infection. RNA was extracted at day 4 of culture for globin gene expression analysis. HNT-34 cells were induced toward the erythroid lineage with hemin $(50 \mu \mathrm{M})$, and at the same time, 5 -azacytidine was added to the medium $(0,200$, or $500 \mathrm{nM})$ to induce expression of the imprinted genes. HNT-34 cells were analyzed for GPA expression 3 days after induction, and RNA was extracted for analysis of gene expression.

RNA extraction and cDNA synthesis. Cell fractions from patients were lysed in RLT buffer for extraction using the RNeasy kit (Qiagen). Individual patient colonies and clones were picked into $50 \mu \mathrm{l}$ RLT buffer, RNA extractions were performed on $30 \mu \mathrm{l}$ of the lysates using either the RNeasy kit or the PicoPure RNA extraction kit (Arcturus), both according to the manufacturer's instructions, with an additional final spin of the columns while empty before elution. cDNA synthesis was performed as described previously (24). For tammar wallabies, cDNA was generated using random hexamers or oligo dT using Fermentas RevertAid H minus First Strand cDNA Kit. For macaques, cDNA was obtained by RNA extraction using the Qiagen RNeasy kit followed by DNase I treatment and random hexamer reverse transcription as above.

Tammar wallaby SNP genotyping. The sequence for the tammar wallaby was inferred from the South American short-tailed opossum and confirmed by sequencing the entire L3MBTL1 gene to identify an expressed C/T SNP in 4 animals; no other SNPs were found. See Supplemental Methods for SNP primer sequences.

Macaque SNP genotyping. L3MBTL1 and SGK2 were analyzed in 4 animals ( 2 male and 2 female) and were found to be informative for at least 3 SNPs in each gene. SNPs at chr10 (-) 20910951 (L3MBTL1) and 20867268 (SGK2) were used for further analysis.

Human SNP genotyping for expressed alleles. See Supplemental Methods.

Mutation and sequencing analysis. See Supplemental Methods.

Bisulphite sequencing and quantitative pyrosequencing assay. See Supplemental Methods.

qPCR. Real-time PCR was performed using SYBR green Q-PCR mix (ThermoScientific). For each gene, primer concentrations were optimized when PCR was performed for 40 cycles of 15 seconds at $95^{\circ} \mathrm{C}$, then 45 seconds at $61^{\circ} \mathrm{C}$, to give standard curves between $90 \%$ and $110 \%$ efficiency. Relative values were quantitated using a relative standard curve dilution of 293 T or K562 cell line cDNA. For all samples, qPCR was performed in duplicate for the experimental gene and $A B L$ control. Values were then normalized to the $A B L$ value for each sample cDNA tested. The threshold of acceptable $A B L$ values for samples was less than 34 cycles for colony RNAderived cDNAs and less than 33 cycles for all other cDNAs. See Supplemental Methods for primer sequences.

CDR gene expression analysis. cDNA was prepared from $\mathrm{BM} \mathrm{CD} 34^{+}$cells, taken from hematologically normal individuals less than 60 years old undergoing hip replacement surgery. $\mathrm{CD} 1^{+} \mathrm{GPA}^{+}$erythroid cells, $\mathrm{CD} 2^{+}$ $\mathrm{T}$ cells, granulocytes, and CD45-depleted platelets were isolated from blood donations from hematologically normal individuals. CD45 deple- tion and CD2 selection was performed using immunomagnetic beads following the manufacturer's instructions (Dynabeads; Invitrogen), and CD34 and GPA selection was performed using immunomagnetic beads following the manufacturer's instructions (Miltenyi Biotech). Erythroid cells were prepared using the single-phase erythroid differentiation system, beginning with the peripheral blood buffy coat mononuclear cell step. Cells were analyzed by FACS on day 8 to show that all $\mathrm{GPA}^{+}$cells were also $\mathrm{CD}^{2} 1^{+}$, after which $\mathrm{GPA}^{+}$cells were isolated by magnetic separation. See Supplemental Methods for primer sequences.

Colony genotyping. See Supplemental Methods.

Strand-specific cDNA synthesis and PCR. See Supplemental Methods. ChIP. See Supplemental Methods.

Immunoprecipitation and Western blot. See Supplemental Methods.

Flow cytometry antibodies. CD61-Alexa Fluor 647 (clone VI-PL2) was obtained from Biolegend; all other antibodies were from Invitrogen (Clontech): CD71-APC (clone T56/14), GPA-PE (clone AME-1), CD34-PE-Cy5.5 (clone 581[class III]), and CD41-PE (clone VIPL3). All anti-mouse FACS antibodies were obtained from BD Biosciences - Pharmingen and were described previously (44).

Statistics. Unpaired 2-tailed Student's $t$ test was used for all analyses, using Prism software (version 4; GraphPad). A P value less than 0.05 was considered significant.

Study approval. Studies using human samples were approved by the Cambridge and Eastern Region Ethics Committee (REC; reference no. 07/MRE05/44). Patients gave written informed consent, and research was carried out in accordance with the Declaration of Helsinki. Studies using macaques were approved by the SingHealth IACUC (approval no. 2009/SHS/509). Studies using mice were approved by the UK home office (license nos. 80/2376 and 80/2567). Studies using tammar wallabies were approved by institutional ethics committees at the University of Melbourne (specimen transfer agreement reference no. GB107A).

\section{Acknowledgments}

We are grateful to the staff of Cambridge Blood and Stem Cell Biobank for provision of samples and to Anna Petrunkina for flow cytometry support. We are also indebted to Matthew Hardy, David Kent, Juan Li, Geoff Shaw, Andy Pask, Rita Ferreira, and Nasios Fourouclas for advice and/or technical assistance. The A.R. Green laboratory is supported by Leukemia and Lymphoma Research, the Cambridge NIHR Biomedical Research Centre, Cancer Research UK, the Leukemia and Lymphoma Society, and the Cambridge Experimental Cancer Medicine Centre. The A.C. Ferguson-Smith laboratory is supported by the MRC and Wellcome Trust and by $\mathrm{A}^{*}$ STAR. P.J. Campbell is a Wellcome Trust senior clinical fellow.

Received for publication July 31, 2012, and accepted in revised form February 7, 2013.

Address correspondence to: Anthony R. Green, Department of Hematology, Cambridge Institute for Medical Research, Hills Road, Cambridge CB2 0XY, United Kingdom. Phone: 44.1223.762665; Fax: 44.1223.762670; E-mail: arg1000@cam.ac.uk.
1. Beroukhim R, et al. The landscape of somatic copynumber alteration across human cancers. Nature. 2010;463(7283):899-905.

2. Bignell GR, et al. Signatures of mutation and selection in the cancer genome. Nature. 2010;463(7283):893-898

3. Baxter EJ, et al. Acquired mutation of the tyrosine kinase JAK2 in human myeloproliferative disorders. Lancet. 2005;365(9464):1054-1061.
4. James C, et al. A unique clonal JAK2 mutation leading to constitutive signalling causes polycythaemia vera. Nature. 2005;434(7037):1144-1148.

5. Kralovics R, et al. A gain-of-function mutation of JAK2 in myeloproliferative disorders. N Engl J Med. 2005;352(17):1779-1790.

6. Levine RL, et al. Activating mutation in the tyrosine kinase JAK2 in polycythemia vera, essential thrombocythemia, and myeloid metaplasia with myelofi- brosis. Cancer Cell. 2005;7(4):387-397.

7. Ebert BL, et al. Identification of RPS 14 as a $5 \mathrm{q}-$ syndrome gene by RNA interference screen. Nature. 2008;451(7176):335-339.

8. Liu TX, et al. Chromosome $5 \mathrm{q}$ deletion and epigenetic suppression of the gene encoding alphacatenin (CTNNA1) in myeloid cell transformation. Nat Med. 2007;13(1):78-83.

9. Starczynowski DT, et al. Identification of miR-145 
and miR-146a as mediators of the $5 \mathrm{q}$ - syndrome phenotype. Nat Med. 2010;16(1):49-58.

10. Asimakopoulos FA, Green AR. Deletions of chromosome $20 \mathrm{q}$ and the pathogenesis of myeloproliferative disorders. Br J Haematol. 1996; 95(2):219-226

11. Bench AJ, et al. A detailed physical and transcriptional map of the region of chromosome 20 that is deleted in myeloproliferative disorders and refinement of the common deleted region. Genomics. 1998;49(3):351-362.

12. Bench AJ, et al. Chromosome 20 deletions in myeloid malignancies: reduction of the common deleted region, generation of a PAC/BAC contig and identification of candidate genes. UK Cancer Cytogenetics Group (UKCCG). Oncogene. 2000;19(34):3902-3913.

13. Bench AJ, et al. Characterization of the imprinted polycomb gene L3MBTL, a candidate $20 \mathrm{q}$ tumour suppressor gene, in patients with myeloid malignancies. Br J Haematol. 2004;127(5):509-518.

14. Boccuni P, MacGrogan D, Scandura JM, Nimer $\mathrm{SD}$. The human $\mathrm{L}(3) \mathrm{MBT}$ polycomb group protein is a transcriptional repressor and interacts physically and functionally with TEL (ETV6). J Biol Chem. 2003;278(17):15412-15420.

15. Sims JK, Rice JC. PR-Set7 establishes a repressive trans-tail histone code that regulates differentiation. Mol Cell Biol. 2008;28(14):4459-4468.

16. Trojer P, Li G, Sims RJ. L3MBTL1, a histonemethylation-dependent chromatin lock. Cell. 2007;129(5):915-928

17. Gateff E, Loffler T, Wismar J. A temperaturesensitive brain tumor suppressor mutation of Drosophila melanogaster: developmental studies and molecular localization of the gene. Mech Dev. 1993;41(1):15-31.

18. Wismar J, et al. The Drosophila melanogaster tumor suppressor gene lethal(3)malignant brain tumor encodes a proline-rich protein with a novel zinc finger. Mech Dev. 1995;53(1):141-154.

19. Kim J, et al. Tudor, MBT and chromo domains gauge the degree of lysine methylation. EMBO Rep. 2006;7(4):397-403.

20. Li H, et al. Structural basis for lower lysine methylation state-specific readout by MBT repeats of L3MBTL1 and an engineered PHD finger. Mol Cell. 2007;28(4):677-691.

21. Trojer P, Reinberg D. Beyond histone methyl-lysine binding: how malignant brain tumor (MBT) protein L3MBTL1 impacts chromatin structure. Cell Cycle. 2008;7(5):578-585.

22. Gurvich N, et al. L3MBTL1 polycomb protein, a candidate tumor suppressor in $\operatorname{del}(20 \mathrm{q} 12)$ myeloid disorders, is essential for genome stability. Proc Natl Acad Sci U S A. 2010;107(52):22552-22557.

23. MacGrogan D, et al. Structural integrity and expression of the L3MBTL gene in normal and malignant hematopoietic cells. Genes Chromosomes Cancer. 2004;41(3):203-213.

24. Li J, Bench AJ, Vassiliou GS, Fourouclas N, Ferguson-Smith AC, Green AR. Imprinting of the human L3MBTL gene, a polycomb family member located in a region of chromosome 20 deleted in human myeloid malignancies. Proc Natl Acad Sci U S A. 2004;101(19):7341-7346.

25. Li J, et al. L3mbtl, the mouse orthologue of the imprinted L3MBTL, displays a complex pattern of alternative splicing and escapes genomic imprinting. Genomics. 2005;86(4):489-494.

26. Coan PM, Burton GJ, Ferguson-Smith AC. Imprinted genes in the placenta - a review. Placenta. 2005;26(suppl A):S10-S20.

27. Charalambous M, da Rocha ST, Ferguson-Smith AC. Genomic imprinting, growth control and the allocation of nutritional resources: consequences for postnatal life. Curr Opin Endocrinol Diabetes Obes. 2007;14(1):3-12.
28. Anand S, et al. Effects of the JAK2 mutation on the hematopoietic stem and progenitor compartment in human myeloproliferative neoplasms. Blood. 2011;118(1):177-181

29. Feinberg AP, Tycko B. The history of cancer epigenetics. Nat Rev Cancer. 2004;4(2):143-153.

30. Niemitz EL, et al. Microdeletion of LIT1 in familial Beckwith-Wiedemann syndrome. Am J Hum Genet. 2004;75(5):844-849.

31. Jinno Y, et al. Mosaic and polymorphic imprinting of the WT1 gene in humans. Nat Genet. 1994;6(3):305-309.

32. Killian JK, et al. Mannose 6-phosphate/insulin-like growth factor 2 receptor (M6P/IGF2R) variants in American and Japanese populations. Hum Mutat. 2001;18(1):25-31

33. Nishiwaki K, Niikawa N, Ishikawa M. Polymorphic and tissue-specific imprinting of the human Wilms tumor gene, WT1. Jpn J Hum Genet. 1997; 42(1):205-211.

34. Piras G, et al. Zac1 (Lot1), a potential tumor suppressor gene, and the gene for epsilon-sarcoglycan are maternally imprinted genes: identification by a subtractive screen of novel uniparental fibroblast lines. Mol Cell Biol. 2000;20(9):3308-3315.

35. Plass $\mathrm{C}$, et al. Identification of Grf1 on mouse chromosome 9 as an imprinted gene by RLGS-M. Nat Genet. 1996;14(1):106-109.

36. Beillard E, et al. Evaluation of candidate control genes for diagnosis and residual disease detection in leukemic patients using 'real-time' quantitative reverse-transcriptase polymerase chain reaction (RQ-PCR) - a Europe against cancer program. Leukemia. 2003;17(12):2474-2486.

37. Tsai CE, Lin SP, Ito M, Takagi N, Takada S, Ferguson-Smith AC. Genomic imprinting contributes to thyroid hormone metabolism in the mouse embryo. Curr Biol. 2002;12(14):1221-1226.

38. Yevtodiyenko A, Carr MS, Patel N, Schmidt JV. Analysis of candidate imprinted genes linked to Dlk1-Gtl2 using a congenic mouse line. Mamm Genome. 2002;13(11):633-638.

39. Messerschmidt DM, de Vries W, Ito M, Solter D, Ferguson-Smith A, Knowles BB. Trim28 is required for epigenetic stability during mouse oocyte to embryo transition. Science. 2012;335(6075):1499-1502.

40. Evans HK, Wylie AA, Murphy SK, Jirtle RL. The neuronatin gene resides in a "micro-imprinted" domain on human chromosome $20 \mathrm{q} 11.2$. Genomics. 2001;77(1-2):99-104.

41. Dupont S, et al. The JAK2 $617 \mathrm{~V}>\mathrm{F}$ mutation triggers erythropoietin hypersensitivity and terminal erythroid amplification in primary cells from patients with polycythemia vera. Blood. 2007;110(3):1013-1021.

42. Jacob B, et al. Stem cell exhaustion due to Runx1 deficiency is prevented by Evi5 activation in leukemogenesis. Blood. 2009;115(8):1610-1620.

43. Kvinlaug BT, et al. Common and overlapping oncogenic pathways contribute to the evolution of acute myeloid leukemias. Cancer Res. 2011; 71(12):4117-4129.

44. Li J, et al. JAK2 V617F impairs hematopoietic stem cell function in a conditional knock-in mouse model of JAK2 V617F-positive essential thrombocythemia. Blood. 2010;116(9):1528-1538.

45. Motoda L, et al. Runx1 protects hematopoietic stem/progenitor cells from oncogenic insult. Stem Cells. 2007;25(12):2976-2986.

46. Petzer AL, Eaves CJ, Barnett MJ, Eaves AC. Selective expansion of primitive normal hematopoietic cells in cytokine-supplemented cultures of purified cells from patients with chronic myeloid leukemia. Blood. 1997;90(1):64-69.

47. Schemionek M, et al. BCR-ABL enhances differentiation of long-term repopulating hematopoietic stem cells. Blood. 2010;115(16):3185-3195.

48. Mizuno H, Nishida E. The ERK MAP kinase path- way mediates induction of SGK (serum- and glucocorticoid-inducible kinase) by growth factors. Genes Cells. 2001;6(3):261-268

49. Brunet A, Park J, Tran H, Hu LS, Hemmings BA, Greenberg ME. Protein kinase SGK mediates survival signals by phosphorylating the forkhead transcription factor FKHRL1 (FOXO3a). Mol Cell Biol. 2001;21(3):952-965.

50. Chi TH, et al. Sequential roles of Brg, the ATPase subunit of BAF chromatin remodeling complexes, in thymocyte development. Immunity. 2003;19(2):169-182.

51. Trotter KW, Archer TK. Nuclear receptors and chromatin remodeling machinery. Mol Cell Endocrinol. 2007;265-266:162-167.

52. Sif S, Stukenberg PT, Kirschner MW, Kingston RE. Mitotic inactivation of a human SWI/ SNF chromatin remodeling complex. Genes Dev. 1998;12(18):2842-2851.

53. Baker SJ, et al. Chromosome 17 deletions and p53 gene mutations in colorectal carcinomas. Science. 1989;244(4901):217-221.

54. Bookstein R, et al. Promoter deletion and loss of retinoblastoma gene expression in human prostate carcinoma. Proc Natl Acad Sci U S A. 1990;87(19):7762-7766

55. Caldas C, et al. Frequent somatic mutations and homozygous deletions of the p16 (MTS1) gene in pancreatic adenocarcinoma. Nat Genet. 1994;8(1):27-32.

56. Gimelbrant A, Hutchinson JN, Thompson BR, Chess A. Widespread monoallelic expression on human autosomes. Science. 2007;318(5853):1136-1140.

57. Qin J, et al. Chromatin protein L3MBTL1 is dispensable for development and tumor suppression in mice. J Biol Chem. 2010;285(36):27767-27775.

58. Asimakopoulos FA, Gilbert JG, Aldred MA, Pearson TC, Green AR. Interstitial deletion constitutes the major mechanism for loss of heterozygosity on chromosome $20 \mathrm{q}$ in polycythemia vera. Blood. 1996;88(7):2690-2698.

59. Barlow JL, et al. A p53-dependent mechanism underlies macrocytic anemia in a mouse model of human 5q- syndrome. Nat Med. 2009;16(1):59-66.

60. Baylin SB, Jones PA. A decade of exploring the cancer epigenome - biological and translational implications. Nat Rev Cancer. 2011;11(10):726-734.

61. Schaub FX, et al. Clonal analysis of deletions on chromosome 20q and JAK2-V617F in MPD suggests that del $20 \mathrm{q}$ acts independently and is not one of the predisposing mutations for JAK2-V617F. Blood. 2009;113(9):2022-2027.

62. Wajapeyee $N$, et al. Senescence induction in human fibroblasts and hematopoietic progenitors by leukemogenic fusion proteins. Blood. 2010;115(24):5057-5060.

63. Perna F, et al. Depletion of L3MBTL1 promotes the erythroid differentiation of human hematopoietic progenitor cells: possible role in $20 \mathrm{q}$ - polycythemia vera. Blood. 2010;116(15):2812-2821.

64. Reisman D, Glaros S, Thompson EA. The SWI/ SNF complex and cancer. Oncogene. 2009; 28(14):1653-1668.

65. Wilson BG, Roberts CW. SWI/SNF nucleosome remodellers and cancer. Nat Rev Cancer. 2011;11(7):481-492.

66. Rui L, et al. Cooperative epigenetic modulation by cancer amplicon genes. Cancer Cell. 2010; 18(6):590-605.

67. Jayapal SR, Lee KL, Ji P, Kaldis P, Lim B, Lodish HF. Down-regulation of Myc is essential for terminal erythroid maturation. J Biol Chem. 2010;285(51):40252-40265.

68. Macaulay IC, et al. Comparative gene expression profiling of in vitro differentiated megakaryocytes and erythroblasts identifies novel activatory and inhibitory platelet membrane proteins. Blood. 2007;109(8):3260-3269. 